\title{
Principal components of functional connectivity: A new approach to study dynamic brain connectivity during rest
}

\author{
Nora Leonardi a,b ${ }^{\text {a Jonas Richiardi }}{ }^{\text {c,d }}$, Markus Gschwind ${ }^{\text {d,e,f }}$, Samanta Simioni ${ }^{\text {e }}$, Jean-Marie Annoni ${ }^{\text {d,f,g }}$, \\ Myriam Schluep ${ }^{\mathrm{e}}$, Patrik Vuilleumier ${ }^{\mathrm{d}, \mathrm{f}}$, Dimitri Van De Ville ${ }^{\mathrm{a}, \mathrm{b}, *}$ \\ a Institute of Bioengineering, Ecole Polytechnique Fédérale de Lausanne (EPFL), Lausanne, Switzerland \\ b Department of Radiology and Medical Informatics, University of Geneva, Geneva, Switzerland \\ c Department of Neurology and Neurological Sciences, Stanford University, Stanford, USA \\ d Department of Neuroscience, University Medical Center (CMU), University of Geneva, Geneva, Switzerland \\ e Department of Clinical Neurosciences, Centre Hospitalier Universitaire Vaudois (CHUV) and University of Lausanne, Lausanne, Switzerland \\ ${ }^{\mathrm{f}}$ Department of Neurology, Hopitaux Universitaires de Genève (HUG), Geneva, Switzerland \\ ${ }^{\mathrm{g}}$ Neurology Unit, University of Fribourg, Fribourg, Switzerland
}

\section{A R T I C L E I N F O}

Article history:

Accepted 5 July 2013

Available online 18 July 2013

\section{Keywords:}

fMRI

Resting state

Dynamics

Functional connectivity

Multiple sclerosis

\begin{abstract}
A B S T R A C T
Functional connectivity (FC) as measured by correlation between fMRI BOLD time courses of distinct brain regions has revealed meaningful organization of spontaneous fluctuations in the resting brain. However, an increasing amount of evidence points to non-stationarity of FC; i.e., FC dynamically changes over time reflecting additional and rich information about brain organization, but representing new challenges for analysis and interpretation. Here, we propose a data-driven approach based on principal component analysis (PCA) to reveal hidden patterns of coherent FC dynamics across multiple subjects. We demonstrate the feasibility and relevance of this new approach by examining the differences in dynamic FC between 13 healthy control subjects and 15 minimally disabled relapse-remitting multiple sclerosis patients. We estimated whole-brain dynamic FC of regionally-averaged BOLD activity using sliding time windows. We then used PCA to identify FC patterns, termed "eigenconnectivities", that reflect meaningful patterns in FC fluctuations. We then assessed the contributions of these patterns to the dynamic FC at any given time point and identified a network of connections centered on the default-mode network with altered contribution in patients. Our results complement traditional stationary analyses, and reveal novel insights into brain connectivity dynamics and their modulation in a neurodegenerative disease.
\end{abstract}

(c) 2013 Elsevier Inc. All rights reserved.

\section{Introduction}

Spontaneous fluctuations of the functional MRI (fMRI) bloodoxygen-level-dependent (BOLD) signal are not random but temporally coherent between distinct brain regions. While these fluctuations were long considered as "noise", Biswal et al. (1995) showed that fluctuations of motor areas were correlated even in the absence of a motor task. Several other networks of coherent BOLD activity between remote

Abbreviations: ACC, anterior cingulate gyrus; BOLD, blood-oxygen-level-dependent; DMN, default mode network; EDSS, Expanded Disability Status Scale; EEG, electroencephalography; FC, functional connectivity; fMRI, functional magnetic resonance imaging; HC, healthy control; ICA, independent component analysis; IFG, inferior frontal gyrus; IPG inferior parietal gyrus; MFG, middle frontal gyrus; MTG, middle temporal gyrus; MTP, middle temporal pole; Paracen, paracentral gyrus; PCA, principal component analysis; PCC, posterior cingulate gyrus; PreC, precuneus; Precen, precentral gyrus; RolOp, rolandic operculum; RRMS, relapse-remitting multiple sclerosis; SD, standard deviation; SMA, supplementary motor area; SFG, superior frontal gyrus; SPG, superior parietal gyrus; SupMarg, supramarginal gyrus; SVD, singular value decomposition.

* Corresponding author at: EPFL/IBI-STI/GRVDV, Station 17, 1015 Lausanne, Switzerland. E-mail address: dimitri.vandeville@epfl.ch (D. Van De Ville). brain regions have since been identified, including visual, auditory, language and attention networks, and a network called the "default mode network" (DMN) which reduces its activity during attentiondemanding tasks. These networks of regions with coherent activity during rest are consistent across subjects and closely resemble the brain's functional organization of evoked responses (Damoiseaux et al., 2006; Fox and Raichle, 2007; Laird et al., 2011; Smith et al., 2009). Coherent BOLD activity persists during sleep and in anesthetized monkeys, suggesting that it reflects a fundamental property of the brain's functional organization (Larson-Prior et al., 2009; Vincent et al., 2007).

Coherent BOLD activity, known as "functional connectivity" (FC), is modulated by learning (Bassett et al., 2011), cognitive and affective states (Cribben et al., 2012; Ekman et al., 2012; Eryilmaz et al., 2011; Richiardi et al., 2011; Shirer et al., 2012) and also spontaneously (Britz et al., 2010; Chang and Glover, 2010; Kitzbichler et al., 2009). Chang and Glover (2010) showed that FC between the posterior cingulate cortex, a key region of the default mode network, and various other brain regions was highly dynamic over time. Standard stationary FC analyses assume temporal stationarity and are blind to the temporal evolution of FC. Several recent studies have since confirmed the non-stationarity of 
FC (Handwerker et al., 2012; Hutchison et al., in press; Kang et al., 2011; Kiviniemi et al., 2011; Li et al., in press; Majeed et al., 2011). We will use the terms dynamic FC to specifically refer to fluctuating connectivity during rest, and stationary FC to refer to connectivity estimated under the assumption of temporal stationarity. Chang et al. (2013b) reported a link between heart rate variability and dynamic FC in some brain regions related to arousal and vigilance, suggesting a neural basis for these fluctuations, and Hutchison et al. (in press) showed that these fluctuations persist in anesthetized monkeys in the absence of conscious, cognitive processing.

When no temporal stationarity is assumed, whole-brain and multisubject FC studies get vastly more complex. Also, given that there is no task, dynamic FC patterns must be learned from the data. Due to these challenges many studies assessing FC changes have focused on individual brain regions or networks, such as the extensively studied DMN. Allen et al. (in press) recently proposed a data-driven approach based on $k$-means clustering to identify whole-brain "FC states" in the resting brain. The authors showed that these states differed strongly from FC patterns identified in stationary analyses and suggested that they represent departures from average FC patterns as FC between individual brain regions appears or disappears. They also highlighted the benefit of whole-brain studies to understand the dynamic interplay between different brain regions or networks.

Given the increasing evidence of dynamic FC during rest and its importance for characterizing the brain's intrinsic functional organization, the aim of our study was to develop a data-driven technique to reveal characteristic fluctuations of whole-brain FC. Our method exploits whole-brain FC of multiple subjects to identify connectivity patterns with coherent dynamics and their contributions across time. Specifically, we estimated dynamic FC by calculating the correlations between the BOLD activity of anatomically defined brain regions covering the whole brain using sliding time windows (Chang and Glover, 2010). We then temporally concatenated the dynamic FC between all pairs of brain regions across subjects to construct a connectivity $\times$ time-subject data matrix. We then applied principal component analysis (PCA), which finds intrinsic structure in the data that here represents patterns of coherent FC dynamics. Using these FC patterns, termed "eigenconnectivities", the FC at any specific time can be represented as a weighted sum. Thus, we can interpret the eigenconnectivities as building blocks of dynamic FC with a contribution that varies across time. In contrast to the clustering approach employed in electroencephalography (EEG) microstate (Pascual-Marqui et al., 1995) and FC state analysis (Allen et al., in press), where stable EEG scalp topographies or wholebrain FC states are identified, we used PCA to identify the most abundant building blocks that reappear across time and subjects.

PCA has previously been applied by Friston et al. $(1993,1994)$ to voxel-wise PET and fMRI data to determine patterns of correlated brain activity, so-called "eigenimages". In contrast to our work, the authors applied PCA to activity and not connectivity time courses, and to single subjects only, which complicates comparisons across subjects. More commonly, voxels with similar resting state activity are identified using spatial independent component analysis (ICA), which decomposes a voxel $\times$ time-subject data matrix into (maximally independent) spatial maps and associated time courses (Kiviniemi et al., 2003; McKeown et al., 1998). The application of PCA and ICA in these studies, however, implicitly assumes FC to be temporally stationary and identifies patterns of voxels with similar resting state activity across the entire scan.

Singular value decomposition ${ }^{1}$ (SVD) has also been applied to uncover patterns of relations between brain activity and experimental

\footnotetext{
1 There is a well-known relationship between SVD and PCA: for centered data $\mathbf{X}$, the left singular vectors of SVD (which is applied to $\mathbf{X}$ ) equal the principal components of PCA (which is applied to the covariance matrix $\mathbf{X} \mathbf{X}^{T}$ ): $\mathbf{X}=\mathbf{U} \Sigma \mathbf{V}^{T} \rightarrow \mathbf{X} \mathbf{X}^{T}=\mathbf{U} \Sigma \mathbf{V}^{T}\left(\mathbf{U} \Sigma \mathbf{V}^{T}\right)^{T}=$ $\mathbf{U} \Sigma \mathbf{V}^{T} \mathbf{V} \Sigma \mathbf{U}=\mathbf{U} \Sigma^{2} \mathbf{U}^{T}$ since $\mathbf{V}^{T} \mathbf{V}=\mathbf{I}$. The columns of $\mathbf{U}$ are called left singular vectors or principal components.
}

conditions, behavior or the activity of other voxels in a seed region, in a method called Partial Least Squares (PLS) (Krishnan et al., 2011; McIntosh et al., 1996). The right singular vectors contain patterns of voxels that are associated with e.g. reaction time or seed voxel's activity (i.e., functionally connected). Here, rather than finding relations between the brain activities of different voxels across different conditions, we identify relations between brain connectivity time courses in an unsupervised manner.

Stationary FC analysis during rest has been widely applied to understand the functional impact of neurological and psychiatric diseases, such as Alzheimer's disease or schizophrenia, and to search for reliable biomarkers (Fox and Greicius, 2010). To demonstrate the feasibility and relevance of the proposed building blocks, we are interested in early dynamic FC changes in patients with multiple sclerosis (MS). MS is an inflammatory disease that attacks the myelin sheaths in the central nervous system, causing brain lesions, which are randomly distributed in periventricular white matter, but concentrated around the frontal and occipital horns of the lateral ventricle, and at the corticosubcortical junction (Compston and Coles, 2008). This was also the case in our minimally disabled relapsing-remitting patients (RRMS) group. MS is usually accompanied by sensory or motor deficits and cognitive impairment, but the correlation between clinical disability and focal lesions visible in structural MRI is poor (Barkhof, 2002; Filippi and Agosta, 2010). This is because "hidden" damage occurs in normal appearing brain tissue (NABT), extending well beyond focal lesions (Fu et al., 1998; Hackmack et al., 2012). Alternative imaging methods, such as resting-state fMRI, have started to address the issue of hidden damage and shown disrupted stationary FC. While some studies restricted their analysis to the DMN or motor regions (Bonavita et al., 2011; Dogonowski et al., 2013; Lowe et al., 2002; Rocca et al., 2010), others have reported changes in several large-scale resting state networks, including the sensorimotor and executive control networks (Hawellek et al., 2011; Richiardi et al., 2012; Rocca et al., 2012; Roosendaal et al., 2010). Together, these results are very promising for characterizing the functional pathology of MS, but the relationship between structural damage and functional changes is still poorly understood (Schoonheim and Filippi, 2012).

Here, we study a group of minimally disabled RRMS patients and demonstrate for the first time how dynamic FC is altered in these patients. Specifically, we examine whether the contribution of the identified FC patterns is disturbed in the early stage of MS. We believe that the study of dynamic FC can further contribute to elucidating functional adaptation and reorganization in this pathology.

\section{Methods}

\section{Subjects}

Subjects and MRI data were the same as in a previous study of our group (Richiardi et al., 2012). RRMS patients were diagnosed according to McDonald's criteria (Polman et al., 2005) and selected using criteria for 1) mild to moderate neurological disability with no impaired ambulation (Expanded Disability Status Scale (EDSS) $\leq 2.5$ (Kurtzke, 1983)), 2) no clinical relapse or corticosteroid therapy during the 6 weeks before inclusion in the study, and 3) no other neurological or psychiatric illness according to DSM-IV criteria. 22 RRMS patients (mean \pm SD age $36.8 \pm 8$ years, 14 females, EDSS $1.9 \pm 0.4$, disease duration $4.7 \pm$ 3.5 years) and 14 healthy control (HC) subjects (age $38.4 \pm 6$ years, 9 females) were included. The study was approved by the local institutional ethics committee and all subjects provided informed consent.

\section{Data acquisition}

Data were acquired on a Siemens 3T TrioTIM using a 32-channel head coil. Functional imaging data were acquired in one session using gradientecho planar imaging $\left(\mathrm{TE}=27 \mathrm{~ms}, \mathrm{TR}=1.1 \mathrm{~s}\right.$, flip angle $=90^{\circ}$, 
matrix $=64 \times 64,21$ transverse slices, voxel size $=3.75 \times 3.75 \times$ $5.63 \mathrm{~mm}^{3}, 450$ volumes). Subjects were instructed to lie still with their eyes closed and not to fall asleep (which was confirmed in a debriefing after the scan).

T1 anatomical scans were collected using an MPRAGE sequence $\left(\mathrm{TE}=2.98 \mathrm{~ms}, \mathrm{TR}=2.4 \mathrm{~s}\right.$, flip angle $=9^{\circ}, 160$ slices, voxel size $=$ $1 \times 1 \times 1.2 \mathrm{~mm}^{3}$ ).

\section{Data preprocessing}

Anatomical and functional data were preprocessed using SPM8 (www.fil.ion.ucl.ac.uk/spm), and a combination of in-house MATLAB scripts and scripts from the DPARSFA toolbox (Chao-Gan and Yu-Feng, 2010). The first ten functional volumes were discarded to allow for T1 equilibration effects. The other 440 functional volumes were spatially realigned to the mean image, detrended (linear and quadratic trends) and bandpass filtered $(0.01-0.15 \mathrm{~Hz})$. To further minimize spurious changes in connectivity related to motion, we "scrubbed" our data by removing all volumes that either 1) had a frame-wise displacement $F D=|\Delta x|+|\Delta y|+|\Delta z|+|\Delta \alpha|+|\Delta \beta|+|\Delta \gamma|>0.5$, where the rotational displacements $\alpha, \beta, \gamma$ were converted to millimeters by calculating the displacement on the surface of a sphere of radius $50 \mathrm{~mm}$ (Power et al., 2012), or 2) were considered as intensity spikes, defined as a whole-brain intensity of more than 2 standard deviations from the average intensity across the duration of the scan. We also excluded the preceding 2 and following 4 volumes for both indices, corresponding to a similar temporal coverage as in Power et al. (2012). Because of the sensitivity of functional connectivity to motion and to retain sufficient data for the dynamic connectivity analysis, we excluded $1 \mathrm{HC}$ subjects and 7 RRMS patients with either maximal head motion above $3 \mathrm{~mm}$ or $2^{\circ}$, or more than 10 out of 440 volumes marked for scrubbing (before adding the 6 surrounding volumes). All results thus reflect a total of 13 HC subjects and 15 RRMS patients.

The functional data were coregistered with each subject's anatomical data. The anatomical data were then segmented (new segmentation algorithm of SPM8, an extension of the unified segmentation algorithm (Ashburner and Friston, 2005)) and regionally parcellated using the automated anatomical labeling (AAL) atlas, which divided the brain into 90 anatomical regions of interest (Supplementary Table 1) (TzourioMazoyer et al., 2002). The segmentation step provided a deformation field, which was used to warp the structural atlas to the subject's native space. We estimated regional mean time series by averaging the fMRI signal over all voxels in each brain region. The time series from the bilateral globus pallidus were discarded due to ventral signal dropout in some subjects, leaving 88 brain regions. We regressed out nuisance variables from the regional time series ( 6 head motion parameters, average cerebrospinal fluid from ventricular masks and white matter signal from white matter masks). The global signal was not regressed out as this step is currently debated in the literature.

\section{Dynamic FC estimation}

We estimated dynamic FC by calculating the pairwise Pearson correlation between the residuals of all 88 brain regions using a slidingwindow technique yielding a $88 \times 88$ correlation matrix for each window (Fig. 1a): the sliding-window correlation between the time series $x$ and $y$ was given by $\rho_{x y}(t)=\operatorname{corr}(x[t, t+\Delta t], y[t, t+\Delta t])$, where $\Delta t$ was the window length in TRs and the window was shifted by two TRs for each estimation. Correlations $\rho$ were Fisher r-to- $z$ transformed $(z=\operatorname{atanh}(\rho))$ to make them approximately normally distributed.

We constructed a dynamic FC matrix $\mathbf{C}_{s}$ or each subject, where each column contained a vectorized correlation matrix (Fig. 1b). When vectorizing the correlation matrices, we kept only the upper triangular part due to symmetry, resulting in a $\left(N^{2}-N\right) / 2 \times T_{s}$ matrix, where $N=88$ was the number of brain regions, and $T_{S}$ the number of windows for subject $s$. We normalized each subject's dynamic FC matrix by removing the global mean and dividing by its standard deviation (mean and standard deviation over all time points and FC pairs).

The sliding-window technique has previously been used by several other groups to investigate dynamic FC with window lengths varying from 15 to 120 TRs (Allen et al., in press; Bassett et al., 2011; Chang and Glover, 2010; Handwerker et al., 2012; Hutchison et al., in press) and similar step sizes (Allen et al., in press; Handwerker et al., 2012; Hutchison et al., in press). We used window lengths of 30, 40, 60 and 120 TRs, covering 33, 44, 66 and $132 \mathrm{~s}$ respectively, to investigate the effect of the window length.

\section{Eigenconnectivity estimation}

To estimate characteristic connectivity patterns across time and subjects, we applied PCA to a matrix containing the dynamic FC matrices of all $\mathrm{HC}$ subjects and RRMS patients; i.e. $\mathbf{X}=\left[\mathbf{C}_{1}, \mathbf{C}_{2}, \ldots \mathbf{C}_{S}\right]$. PCA is a statistical technique to detect unknown, yet structured patterns that explain the differences in the collection of vectorized correlation matrices. A few patterns typically explain most of the variation present in all of the original correlation matrices, allowing to automatically summarize the data.

Before applying PCA, the mean of each row is normally subtracted from the data: $\mathbf{X}^{\prime}=\mathbf{X}-\overline{\mathbf{X}}$, where $\overline{\mathbf{X}}$ is a matrix that contains the rowwise means. However, we observed that PCA was partly driven by inter-individual differences in mean correlation levels rather than correlation fluctuations when we subtracted the mean of the concatenated matrix (see the Motion section). Therefore, we subtracted the row-wise means of $\mathbf{C}_{s}$ for each subject $s$ individually before we concatenated all subjects along the temporal dimension: $\mathbf{X}^{\prime}=$ $\left[\mathbf{C}_{1}-\overline{\mathbf{C}}_{1}, \mathbf{C}_{2}-\overline{\mathbf{C}}_{2}, \ldots, \mathbf{C}_{S}-\overline{\mathbf{C}}_{S}\right]$ (Figs. 1b, c).

We then applied PCA, which finds the eigenvectors and eigenvalues of the covariance matrix of $\mathbf{X}^{\prime}$ (Fig. $1 \mathrm{c}$ ): $\mathbf{X}^{\prime} \mathbf{X}^{\prime T}=\mathbf{U} / \mathbf{U}^{T}$, where $\mathbf{U}$ contains orthonormal eigenvectors on its columns and $\Lambda$ the corresponding eigenvalues on its diagonal; i.e., $\Lambda_{k k}=\lambda_{k}$. These eigenvectors (or equally principal components) can be seen as features (or spatial modes) that characterize the variation across the collection of correlation matrices. Because they capture connectivity patterns, we call them "eigenconnectivities".

The matrix $\mathbf{U}$ is of size $\left(N^{2}-N\right) / 2 \times\left(N^{2}-N\right) / 2$; i.e., we obtain $\left(N^{2}-N\right) / 2$ eigenconnectivities. However, the eigenconnectivities with the largest associated eigenvalues explain most of the variation in the collection of dynamic FC matrices and a small number can be used to efficiently approximate the data. Approximating the data using only the $K$ eigenconnectivities with the $K$ largest eigenvalues, results in a retained variance of $\sum_{k=1}^{K} \lambda_{k} / \sum \lambda_{k}$. The retained variance is equal to the approximation accuracy $1-\left\|\mathbf{X}^{\prime}-\mathbf{X}_{\text {approx }}^{\prime}\right\|^{2} /\left\|\mathbf{X}^{\prime}\right\|^{2}$, where $\mathbf{X}_{\text {approx }}^{\prime}=$ $\mathbf{U}_{\text {red }} \mathbf{U}_{\text {red }}^{T} \mathbf{X}^{\prime}$, and $\mathbf{U}_{\text {red }}$ contains only the first $K$ eigenconnectivities.

\section{Time-dependent contribution of eigenconnectivities}

A subject's dynamic FC matrix can be efficiently represented by projecting it onto a few eigenconnectivities: $\mathbf{W}_{s}=\mathbf{U}_{\text {red }}^{T}\left(\mathbf{C}_{s}-\overline{\mathbf{C}}_{s}\right)$, where $\mathbf{W}_{\mathbf{s}}$ is a matrix of size $K \times T_{s}$ containing the weights of $K$ eigenconnectivities at all $T_{s}$ windows (Fig. 1d). These weights are similar to the time-dependent profiles obtained for the "eigenimages" in Friston et al. (1993), and conceptually related to the subject-dependent time courses calculated in independent component analysis (ICA) by dual regression (Beckmann et al., 2009). In ICA, spatial maps from group-ICA are used as regressors to estimate a time course for each independent component and subject. Here, we use the eigenconnectivities obtained from group analysis as regressors to estimate associated time courses for each subject: since the eigenconnectivities are orthonormal we can reformulate the equation above as $\mathbf{C}_{s}-\overline{\mathbf{C}}_{s}=\mathbf{U}_{\text {red }} \mathbf{W}_{s}$, where $\mathbf{U}_{\text {red }}$ acts as the regressor. Thus, for a single set of "group" eigenconnectivities, 
a) Dynamic FC Brain regions

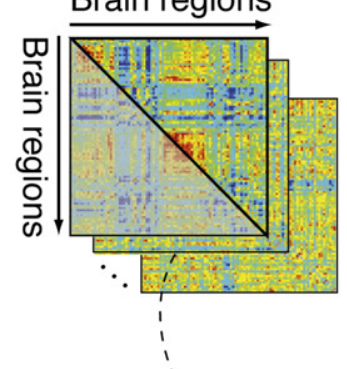

b) Unfolding \&

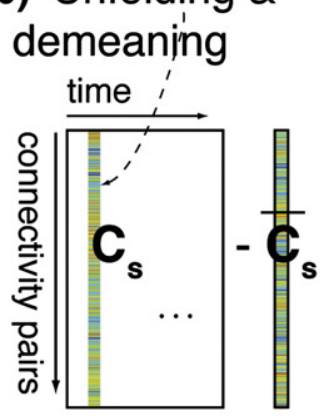

c) Temporal concatenation and estimation of eigenconnectivities

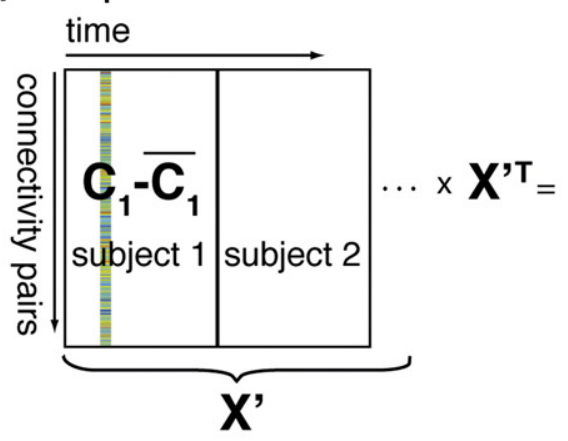

d) Time-dependent weights

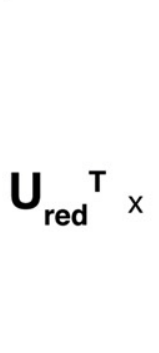

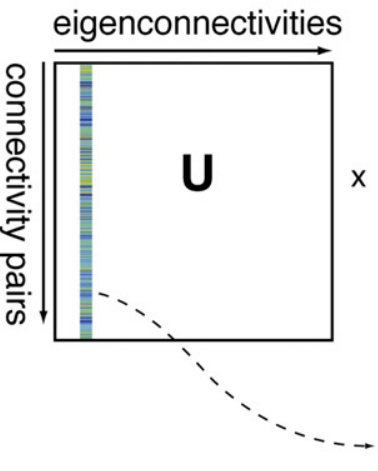

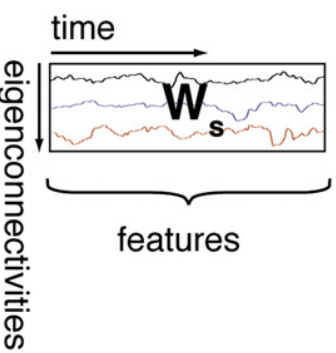

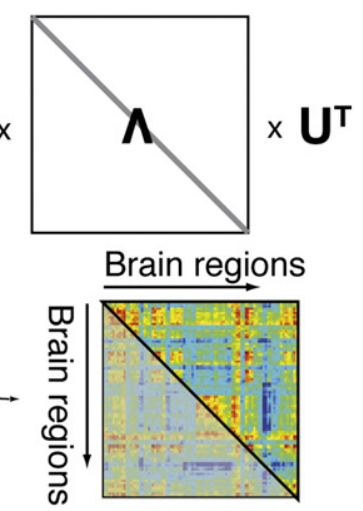

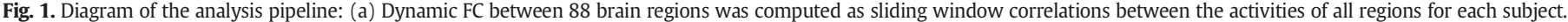

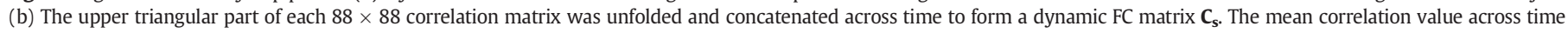

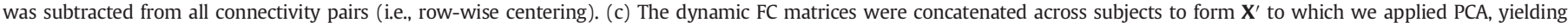

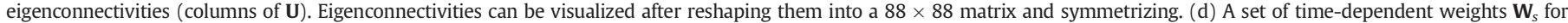
each subject was calculated by projecting the centered dynamic FC matrix onto a few eigenconnectivities.

which we can interpret as building blocks of dynamic FC, we obtain subject-specific time-dependent weights $\mathbf{W}_{s}$ that describe their contribution in representing dynamic FC matrix over time. We inspected the timedependent weights to make sure that the eigenconnectivities contributed to dynamic connectivity in all subjects (i.e., non-zero weights for all subjects).

\section{Comparing HC subjects and RRMS patients}

We first compared the average dynamic FC strength $\overline{\mathbf{C}}_{s}$ between HC subjects and RRMS patients using two-sample t tests. Statistically significant differences were determined using non-parametric permutation testing, by estimating a maximal statistic under the null hypothesis of no difference; i.e., observed differences in the 95th percentile of the null distribution of differences between subjects randomly assigned to the two groups were significant at the $5 \%$ level ( $n=1000$ randomizations, corrected p-value) (Nichols and Holmes, 2002).

We next assessed whether the contribution of the eigenconnectivities was different between HC subjects and RRMS patients by comparing the percentage of time-dependent weights with positive signs. A difference between groups would indicate that the contribution of a specific FC pattern is biased in the RRMS patients. We compared such a summary measure since individual time points are not consistent across subjects in RS studies. We tested for differences in the percentages using Hotelling's $T^{2}$ multivariate test. Hotelling's $T^{2}$ test can also be seen as a maximum univariate $t^{2}$ test for a specific linear combination of the means of the two groups; i.e., the weighted sum $\sum_{k=1}^{K} a_{k}\left(m_{k}^{H C}-m_{k}^{M S}\right)$ is maximal, where $\sum_{k=1}^{K} a_{k}^{2}=1, m_{k}$ is the average percentage for eigenconnectivity $k$ across each group of subjects and $H C$ and MS indicate the HC and RRMS subjects, respectively. Hotelling's $\mathrm{T}^{2}$ test identifies group and interaction (group xeigenconnectivity) effects. For the interaction effect, the weighted sum is modified as $\sum_{k=1}^{K=1} a_{k}\left\{\left(m_{k}^{H C}-m_{k+1}^{H C}\right)-\left(m_{k}^{M S}-m_{k+1}^{M S}\right)\right\}$. We estimated the effect size from the Mahalanobis distance (a multivariate generalization of Cohen's $d$ ), which is defined as: $D=$ $\sqrt{T^{2}\left(1 / n_{1}+1 / n_{2}\right)}$, where $\mathrm{T}^{2}=F K\left(n_{1}+n_{2}-2\right) /\left(n_{1}+n_{2}-K-1\right)$ and $n_{1}, n_{2}$ are the sizes of the HC and RRMS groups and $K=10$ is the number of variables (eigenconnectivities).

We calculated a summary measure of percentage positive weights according to the weights of the Hotelling's $\mathrm{T}^{2}$ test for each subject and assessed whether it was correlated with clinical disability (EDSS) using Pearson correlation within RRMS patients.

\section{Phase randomized data}

Handwerker et al. (2012) showed that the sliding-window technique can result in spurious dynamic FC changes and it is thus crucial to compare the results to an appropriate null model. We phase randomized both brain activity time courses before estimating dynamic FC (Handwerker et al., 2012), and connectivity time courses estimated from original activity (Allen et al., in press) in separate simulations. Specifically, we Fourier transformed each subject's activity or connectivity time courses, randomized the phases independently for each brain region or connectivity pair, and transformed back to the time domain using the amplitude of the real data but randomized phases (Theiler et al., 1992). Phase randomizations preserve the mean, variance and autocorrelation properties of the time courses, while randomizing their precise timing. Fluctuations in FC estimated from phase randomized brain activity are thus due to random activity timing. Randomized connectivity destroys its precise timing, while preserving inter-subject variability in the mean and variance of connectivity (i.e., we can disentangle effects of inter-subject variability in mean FC values from FC fluctuations).

We also assessed the significance of each eigenconnectivity by comparing its eigenvalue to a null distribution obtained using the phase randomized data. Analytic methods to estimate the dimensionality currently employed for activity time courses, such as Laplace PCA (Minka, 
2000), overestimate the dimensionality in the presence of correlated noise (Cordes and Nandy, 2006). Comparing the eigenvalues to a null distribution obtained using surrogate data avoids making parametric assumption about the noise. This approach is similar to how significant latent dimensions are estimated in PLS (Krishnan et al., 2011; McIntosh et al., 1996) and in other fields studying time series (e.g. Bjornsson and Venegas, 1997). The intersection of the real eigenvalue spectrum and the 95th percentile of the phase randomized spectrum estimates the dimensionality of the data ( $n=100$ randomizations, maximal statistic to correct the p-value).

\section{Motion}

As described above, we scrubbed the data and regressed out head motion parameters from the regional time series. However, residual motion may still drive changes in dynamic FC. We thus tested for relationships between the time-dependent weights and four summary measures of motion using Pearson's correlation. We converted FD defined above and root mean square displacement $D=\sqrt{\Delta x^{2}+\Delta y^{2}+\Delta z^{2}}$ to a measure for each window by estimating the intensity (average) and fluctuation of motion (standard deviation) in each window.

Statistically significant correlations with motion were determined using non-parametric testing, similar to above; i.e., observed correlations in the 95th percentile of the null distribution of correlation with phase-randomized motion time series were significant at the $5 \%$ level ( $n=1000$ randomizations, corrected p-value). Note that these phase randomizations were not the same as those used above to obtain a null distribution of dynamic FC.

\section{Results}

\section{Dynamic FC estimation}

After scrubbing, each subject had between 401 and 440 volumes left, from which we estimated dynamic FC matrices $\mathbf{C}_{s}$. For a window length of 30 TRs, the multi-subject data matrix $\mathbf{X}$ was of size $3828 \times 5554$, i.e., it

\section{a) Dynamic FC}

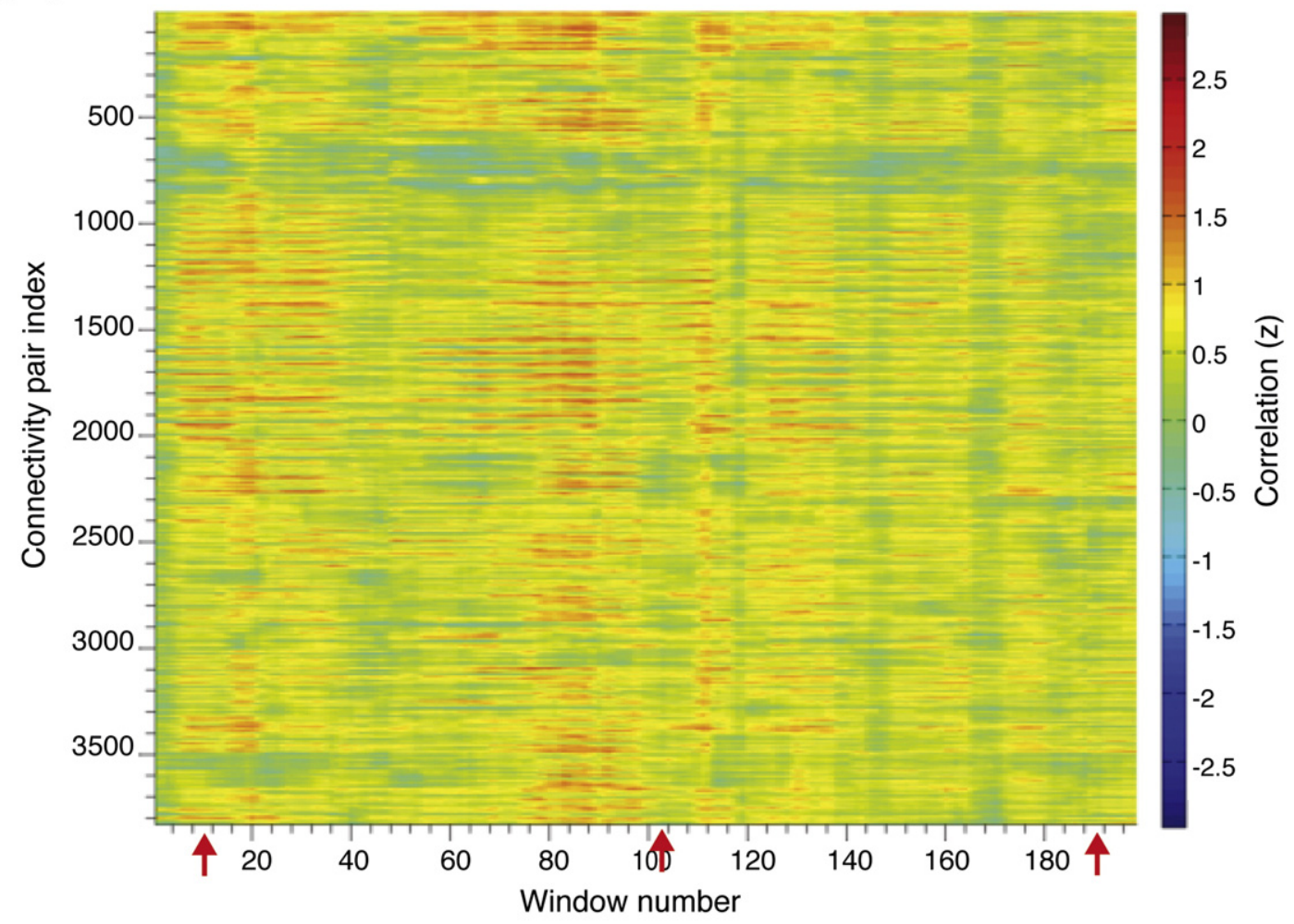

\section{b) Example FC networks}
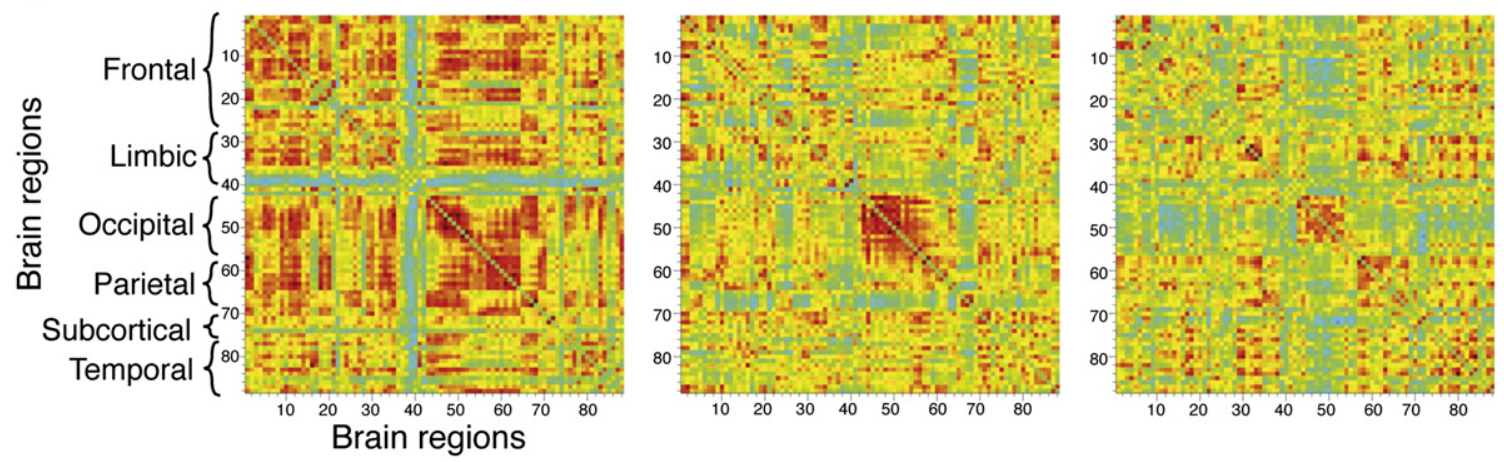

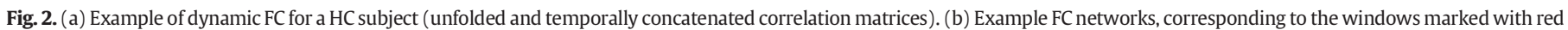
arrows in (a) and reshaped into correlation matrices. 
contained 5554 unfolded correlation matrices across all subjects. Each subject had an average of $\bar{T}=198 \pm 5$ windows and this number did not differ between HC subjects and RRMS patients (two-sample t test, $\mathrm{p}=0.8$ ). Fig. 2 and Supplementary Movie 1 show an example of the estimated dynamic FC for a HC subject. FC is visibly dynamic over the course of the scan as indicated by the change of color across windows.
a)Eigenconnectivity 1
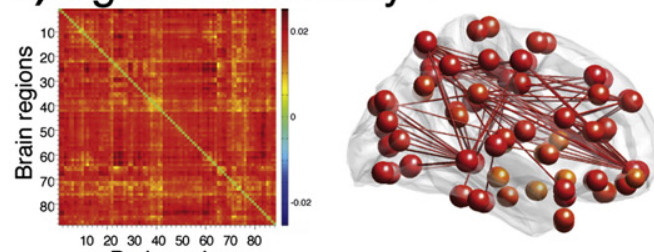

20304050007080
Brain regions

b) Eigenconnectivity 2
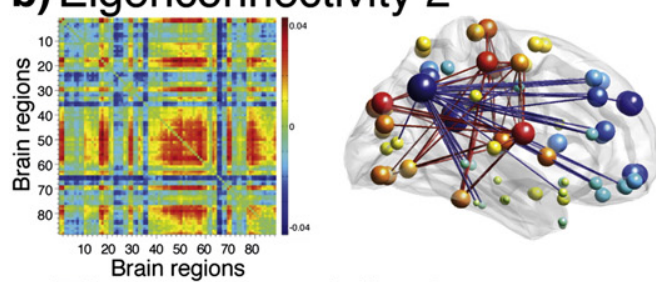

c) Eigenconnectivity 3
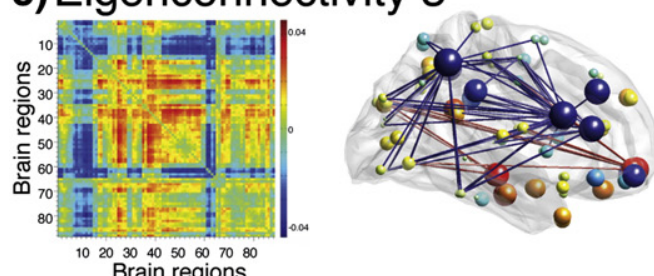

d) Eigenconnectivity 4
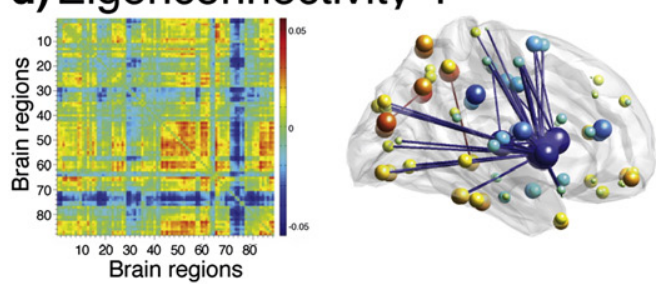

\section{e) Eigenconnectivity 5}
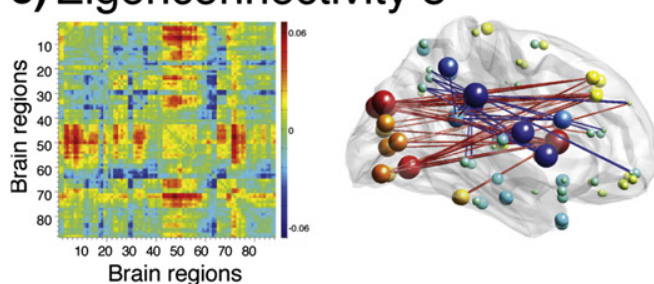

\section{f) Eigenconnectivity 6}
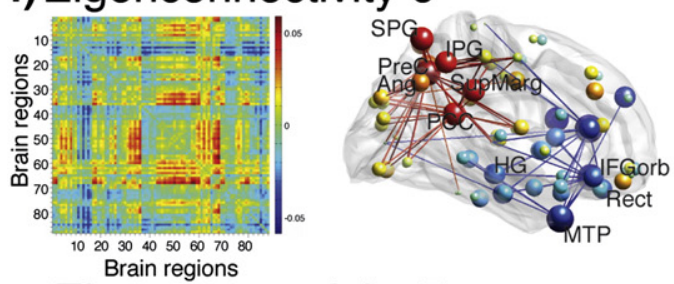

g) Eigenconnectivity 7
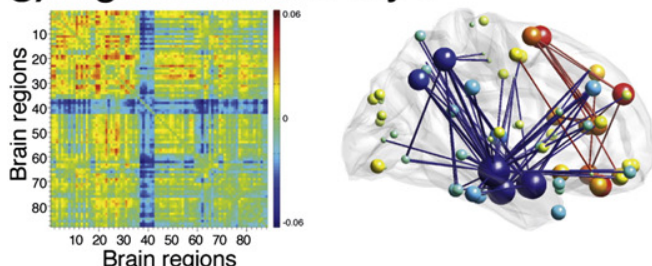

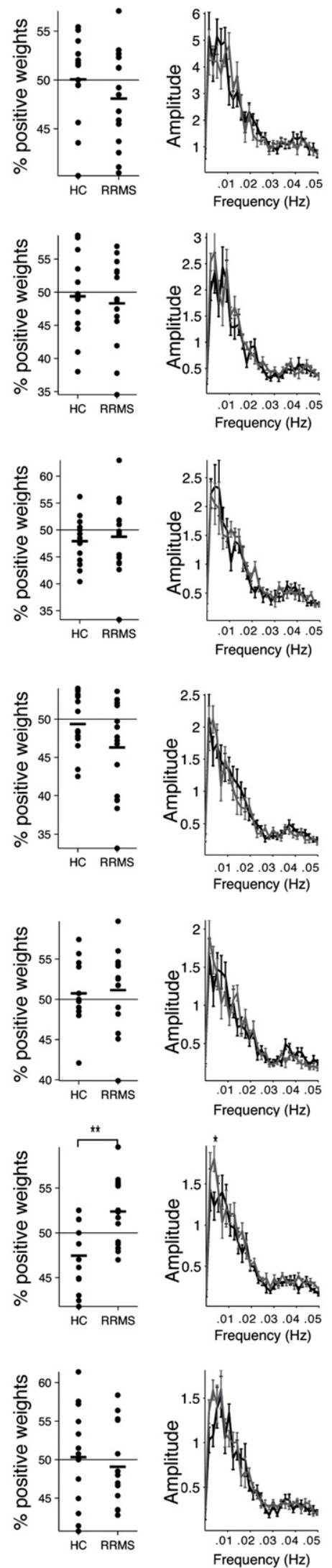
h) Eigenconnectivity 8
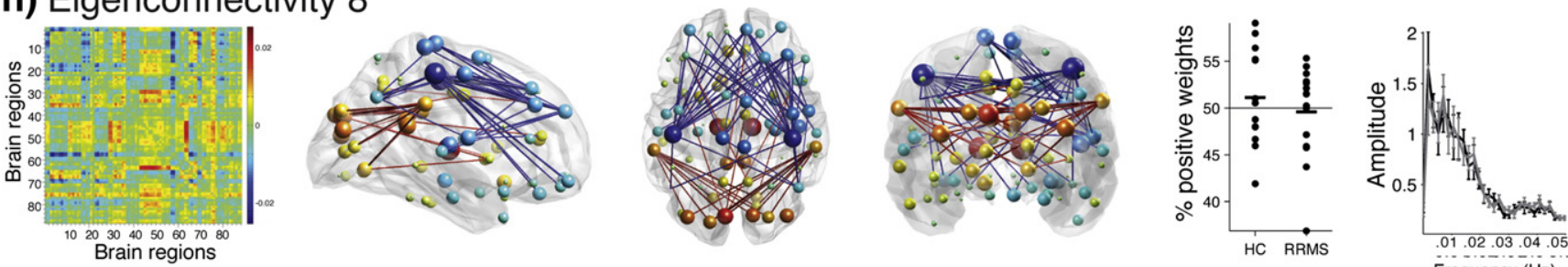

\section{i) Eigenconnectivity 9}
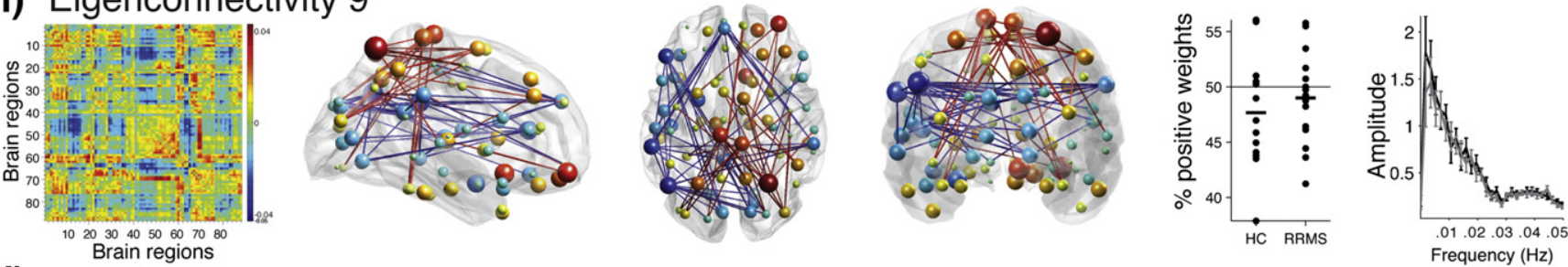

\section{j) Eigenconnectivity 10}
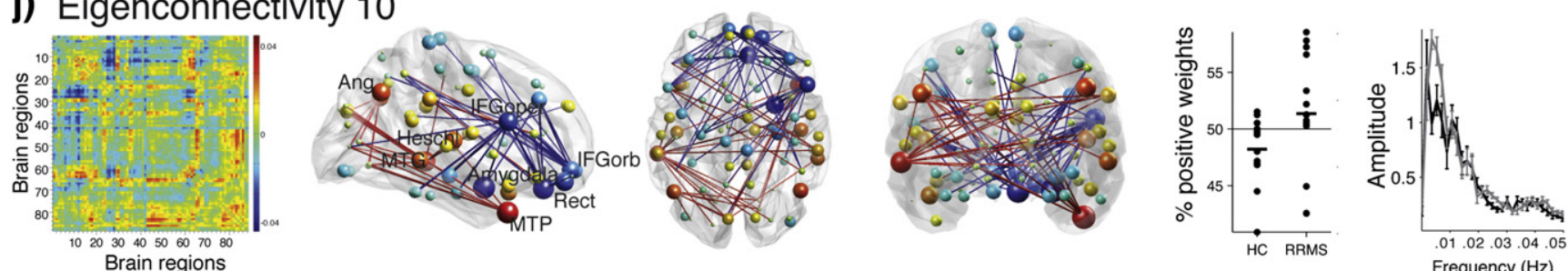

\section{k) Eigenconnectivity 50}

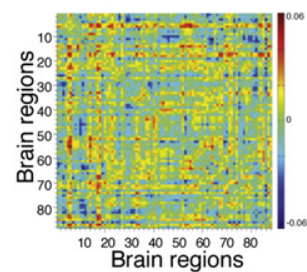

m) Hotelling $T^{2}$ combination

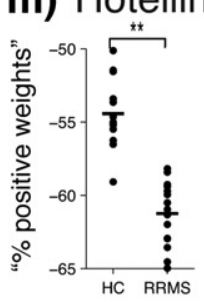

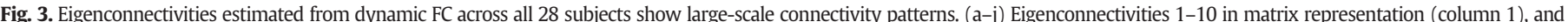

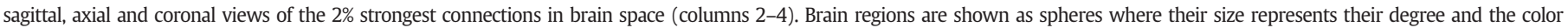

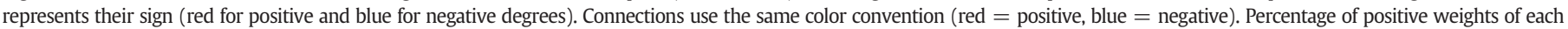

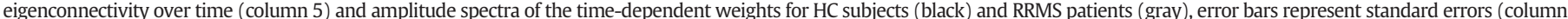

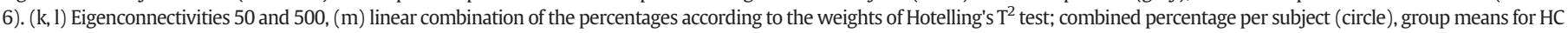
subjects and RRMS patients, respectively (line).

For this subject, the majority of correlations were positive, but we also observed alternations between positive and negative correlations.

\section{Eigenconnectivities}

The average dynamic FC strength $\overline{\mathbf{C}}_{s}$ across each group of subjects showed functional clusters typically observed in stationary FC analyses (Supplementary Fig. 1): strongly connected visual areas (bilateral calcarine, cuneus, lingual gyri and other occipital regions), motor areas (bilateral pre- (Precen) and postcentral gyri, rolandic opercula (RolOp), supplementary area (SMA), paracentral lobule (Paracen)), and regions of the DMN (bilateral superior frontal gyri (SFG), cingulate gyri, angular gyri and precuneus (PreC)).

We then identified dynamic FC patterns across all HC subjects and RRMS patients by calculating the eigenvectors of the covariance matrix of the concatenated, demeaned dynamic FC matrices. The first ten eigenconnectivities retained $34 \%$ of the variance or equally, approximated the data with an accuracy of 34\% (Supplementary Fig. 2 shows all 3828 eigenvalues). The intersection of the real and phase-randomized eigenvalue spectra estimated that the first 30 eigenconnectivities predominantly carried signal ( $5 \%$ corrected significance level, Suppl. Fig. 2). In the remainder of this manuscript we restrict our analysis to the first 10 eigenconnectivities, which have eigenvalues well above the significance level, and because the multivariate Hotelling's $\mathrm{T}^{2}$ test sets an upper limit on the number of components due to group sizes.

Fig. 3 shows some of the estimated "group" eigenconnectivities, which represent orthogonal (independent) connectivity patterns with coherent fluctuations. Since the mean FC strength of each pair has been removed for each subject, these patterns encode how dynamic FC fluctuates around the average connectivity strength over time. The first few eigenconnectivities, which explain most of the variance in the dynamic FC matrices, represent distributed connectivity patterns. Eigenconnectivity 1 identifies an all-positive pattern and reflects overall FC strength ("excursions" around the mean where whole-brain dynamic FC is globally increased or decreased; Fig. 3a): connections between superior frontal regions (23-26) and other regions in the brain were strong (dark red areas) while connections of limbic (38-42) and subcortical regions (71-74) were weaker (yellow areas).

The following eigenconnectivities have both positive and negative patterns (red and blue areas in Fig. 3, respectively). Windows with strong FC in red areas and weak FC in blue areas will have large positive weights for a given eigenconnectivity. Windows with weak FC in red areas and strong FC in blue areas on the other hand will have large negative weights. Therefore, an eigenconnectivity contrasts windows with strong FC in red areas and weak FC in blue areas with those of the opposite character. Also, we can interpret the connectivity patterns as representing connections that covary (i.e., within red areas and within 
blue areas) or anti-covary (i.e., between red and blue areas) according to that dimension of the representation. It should be noted that the sign (i.e., positive and negative for red and blue, respectively) is arbitrary and should not be confounded with anti-correlation for negative signs.

Eigenconnectivity 2 contrasts a network of connections involving mostly regions of the DMN (blue) with regions involved in motion, vision, speech and hearing (red), i.e., it contrasts windows with strong FC in DMN regions with windows with strong FC in sensori-motor regions (Fig. 3b): Blue areas include connections of the medial frontal (23-26), anterior (ACC) and posterior cingulate gyri (PCC; 31, 32, 35, $36)$, angular gyri $(65,66)$ and precuneus $(67,68)$. Red areas include connections of the primary sensori-motor areas $(1,2,18-20,57,58$, $69,70)$, occipital, ventral and dorsal visual areas (43-56, 59, 60), speech $(17,63,64,79,80)$, auditory areas (77-82), and the insula $(29,30)$. Eigenconnectivity 3 identifies a network between regions involved in memory and higher cognitive processing $(25-28,35$, $37-39,67,68)$, the rolandic operculi $(17,18)$ and Heschl's gyri $(77$, 78; red), and another network of connections of the inferior (IFG) and middle frontal gyri (MFG; 7-14), and inferior parietal gyri (IPG; 61, 62; blue; Fig. 3c). Eigenconnectivity 4 identifies a network of connections for thalamic basal ganglia (71-76) and the insula (29, 30; red), and another network of regions involved in visuo-spatial processing (43 - 56, 59 - 62, 83 - 88; blue, Fig. 3d).

Fig. 3 also shows that most FC patterns contain connections of regions situated in both hemispheres, with the exception of eigenconnectivity 6 where the network of blue connections lies predominantly in the left hemisphere (inferior frontal areas) and red ones in the right hemisphere (parietal areas); and eigenconnectivity 9 where most blue connections lie in the left hemisphere.

Eigenconnectivities of higher order, such as e.g. eigenconnectivity 500 , resemble purely artifactual components that do not identify meaningful functional clusters (Fig. 31).

Results with phase-randomized activity or dynamic FC resulted in eigenconnectivities with no apparent structure (Fig. 4, Supplementary Fig. 4e) and little explained variance (5\% for the first ten eigenconnectivities for both randomizations).

\section{Time-dependent contribution of eigenconnectivities}

Fig. 5a shows the time-dependent weights $\mathbf{W}_{s}$ obtained for the same example subject as in Fig. 2 by projecting the dynamic FC matrix onto the first ten eigenconnectivities (see Supplementary Fig. 3 for all subjects). The weights varied smoothly across time but presented pronounced variability, again indicating that FC is highly dynamic. The 5th and 95th percentiles of weights observed for phase-randomized correlation time courses indicate that the observed weights were both within and outside of ranges observed for the surrogate data, and for which time points there is strong evidence for a large contribution of an eigenconnectivity.
The weights of eigenconnectivity 1 (dark blue) track the overall change in FC strength and perfectly correlate with the mean correlation of each window (column-sum of $\mathbf{C}_{s}$ ), $\rho=1, \mathrm{p}<0.001$ for all subjects: at time points when whole-brain dynamic FC is strong, or in other words above average dynamic FC strength (red areas in Fig. 2a), the weight is positive, while at time points when whole-brain dynamic FC is weak (blue areas in Fig. 2a), the weight is negative. The weights of the other eigenconnectivities track more subtle changes in dynamic FC patterns.

Figs. 5b-c show which four eigenconnectivities contributed most strongly to the demeaned example dynamic FC networks of Fig. 2b. The eigenconnectivities with the strongest contributions capture different aspects at a specific window, such as the strong positive occipitoparietal FC and weak FC between limbic regions and the rest of the brain in Fig. 5b.

The weights were highly variable for all subjects, which indicated that the eigenconnectivities captured inherent structure in the fluctuations across all subjects and not just within a single subject.

\section{Comparing HC subjects and RRMS patients}

First, we examined whether the average dynamic FC strength $\overline{\mathbf{C}}_{s}$ differed between HC subjects and RRMS patients. Supplementary Fig. 1 indicates a widespread reduction in FC strength in RRMS patients and two-sample t tests revealed 22 significantly weaker connections, mainly between the left amygdala and occipital and parietal regions; and connections of the middle and posterior cingulate gyri and bilateral superior frontal gyri ( $\mathrm{p}<0.05$, corrected, see Supplementary Table 2 for a full list).

Next, we compared the percentage of positive weights of the ten first eigenconnectivities between HC subjects and RRMS patients (Fig. 3, column 5). HC subjects and RRMS patients differed strongly in the percentage of positive weights, indicating a bias in the contribution of some eigenconnectivities $\left(F_{(10,17)}=4.1, \mathrm{p}=0.005, D=3.0\right.$ for the group effect; $F_{(10,17)}=3.5, \mathrm{p}=0.01, D=2.8$ for the interaction effect; Fig. $3 \mathrm{~m})$. The Hotelling's $\mathrm{T}^{2}$ group difference was $\sum_{k=1}^{K} a_{k}\left(m_{k}^{H C}-\right.$ $\left.m_{k}^{M S}\right)=6.8 \pm 5.3 \%$, with $a_{1}=0.06, a_{2}=0.13, a_{3}=-0.09, a_{4}=$ $0.17, a_{5}=-0.11, a_{6}=-0.72, a_{7}=0.09, a_{8}=0.10, a_{9}=-0.18$, and $a_{10}=-0.63$. The most important differences thus stemmed from eigenconnectivities 6 and 10, which was confirmed in post hoc two-sample $\mathrm{t}$ tests $(\mathrm{p}=0.002, d=-1.29 ; \mathrm{p}=0.06, d=-0.74$, respectively, $\mathrm{p}>0.1$ for the other 8 eigenconnectivities). For both eigenconnectivities RRMS patients had more positive contributions than HC subjects (mean percentage $>50 \%$ ), i.e., their red connections were more often strongly connected (positive weights increase the FC of red areas, and "strongly" here means above average) and their blue ones were more often weakly connected (positive weights reduce the FC of blue areas). Red connections of eigenconnectivity 6 were for example concentrated in posterior regions of the DMN (SPG, PreC, angular gyri, supramarginal gyri (SupMarg)), while blue connections lay mostly

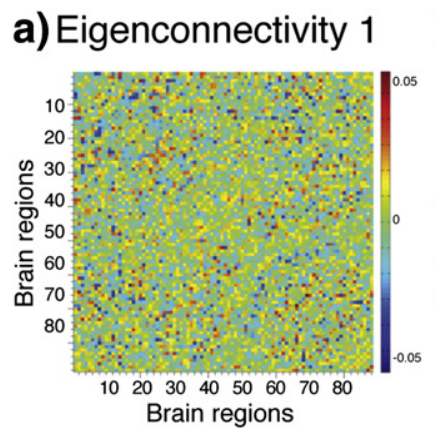

\section{b) Eigenconnectivity 2}

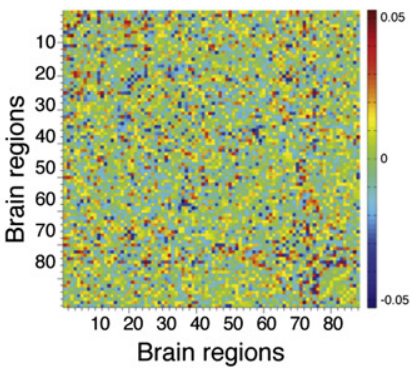

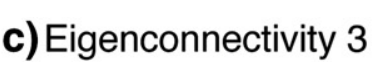

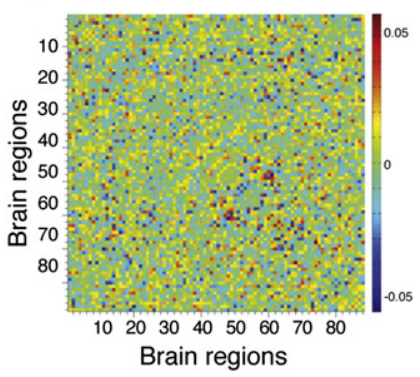

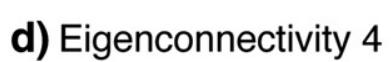

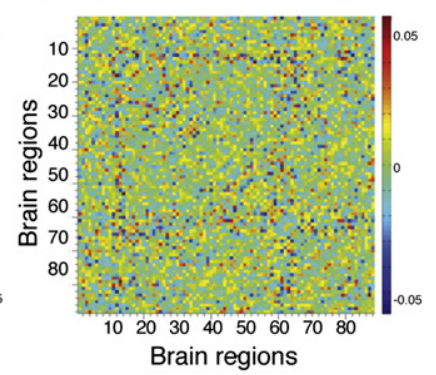

Fig. 4. Eigenconnectivities 1-4 estimated from phase-randomized dynamic FC matrices lose all structure. 


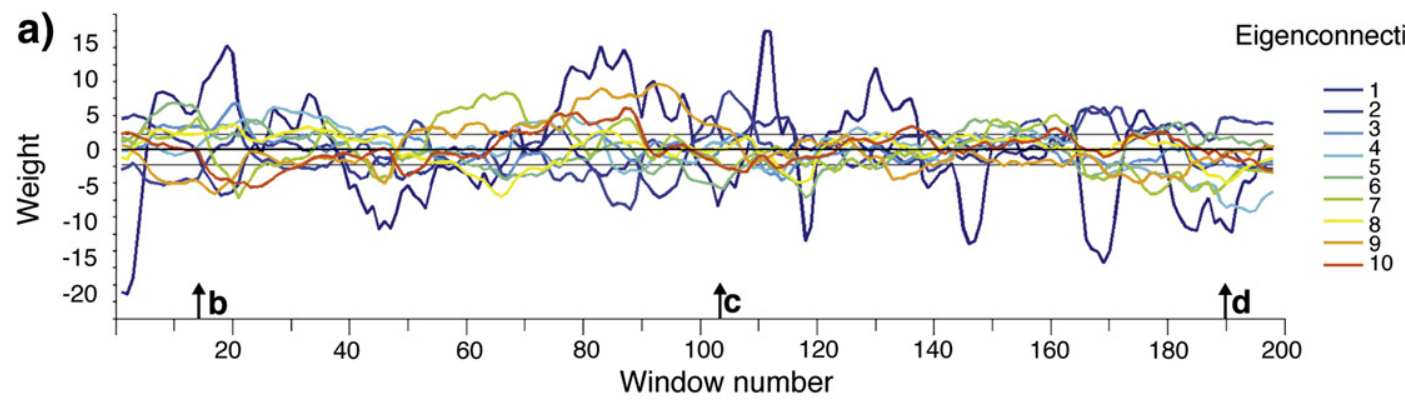

\section{b) Demeaned FC, eigenconnectivities with top 4 contributions}

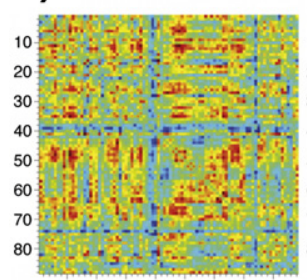

c)
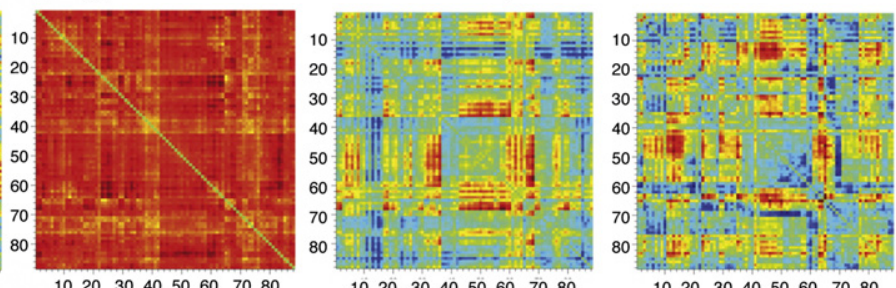

$102030405060 \quad 7080$

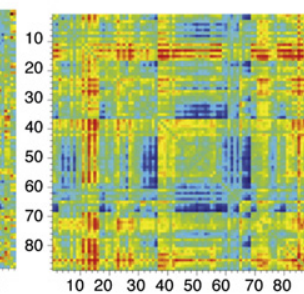

d)
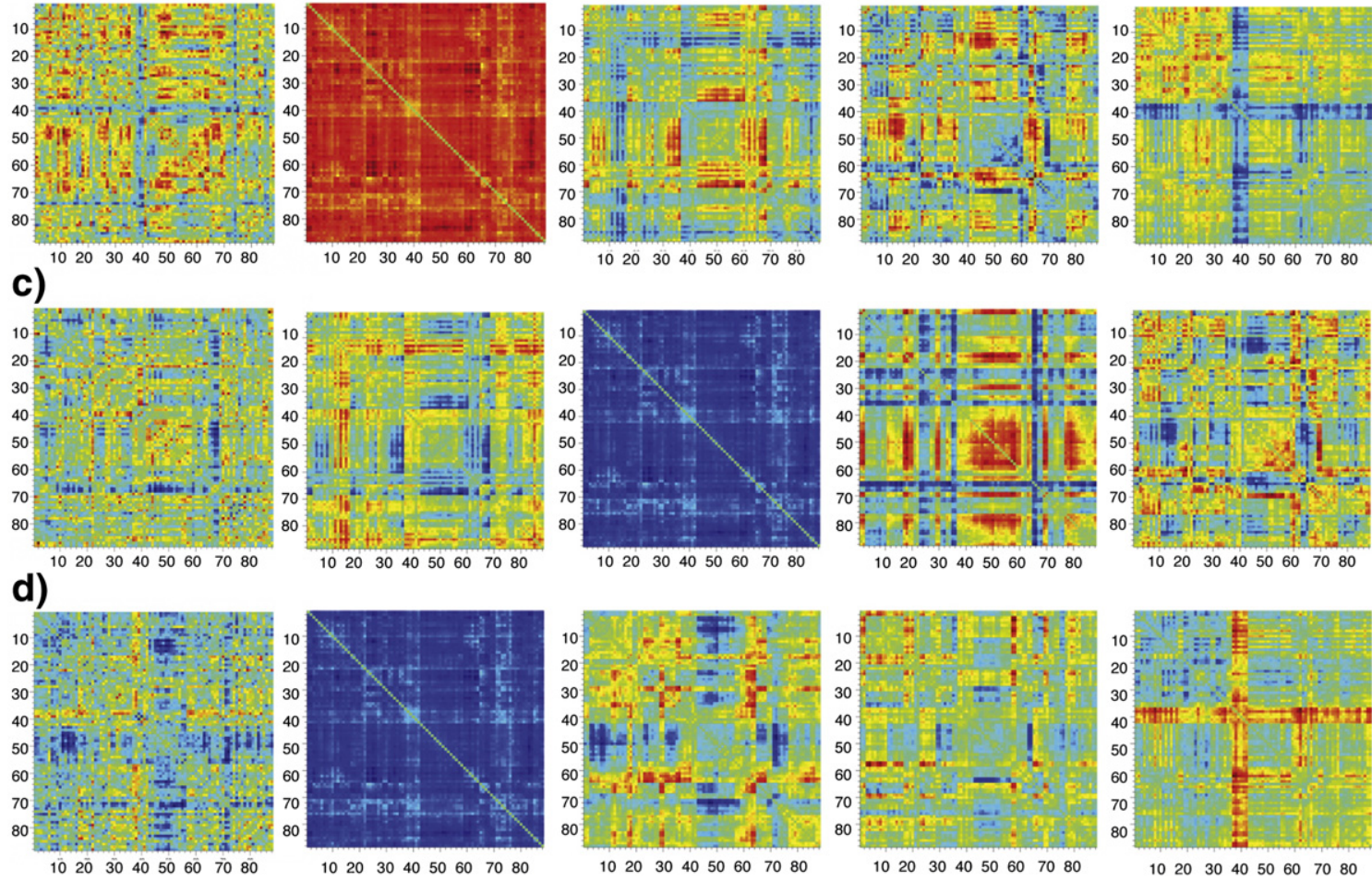

$1020 \quad 30405060 \quad 7080$
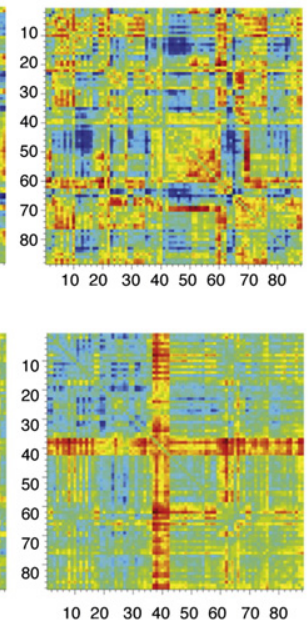

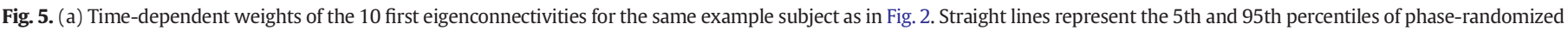

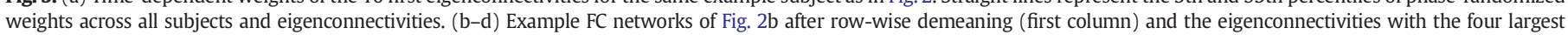

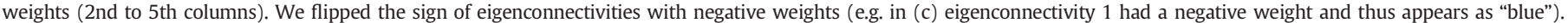
(b), (c) and (d) correspond to the windows marked with arrows in (a).

in anterior (IFG, gyri recti) and midtemporal regions (MTP, Fig. 3f). Red connections of eigenconnectivity 10 included temporal regions (MTP, middle temporal gyrus (MTG), Heschl), and the angular gyri, while blue connections spanned a network of the orbito-frontal regions, right amygdala and motor regions (Precen, Paracen; Fig. 3j). Fig. 3j also shows that many connections in eigenconnectivity 10 were across the hemispheres and ran along the anterior-posterior axis. To explore whether the percentage positive weights were dominated by small weights, we repeated the analysis after excluding the smallest $10 \%$ and $20 \%$ weights and obtained similar results $\left(F_{(10,17)}=4.2, \mathrm{p}=0.005\right.$, $D=3.1$ for the group effect, post hoc tests significant for the sixth $\mathrm{p}=0.006$ and tenth components $\mathrm{p}=0.038$; and $F_{(10,17)}=3.8, \mathrm{p}=$ $0.008, D=2.9$, post hoc tests significant for the sixth $\mathrm{p}=0.02$, and 10th components $\mathrm{p}=0.02$, respectively). The percentage positive weights of the first ten eigenconnectivities for phase-randomized data were not different between HC subjects and RRMS patients (Hotelling's $\mathrm{T}^{2}, \mathrm{p}>0.4$ for both groups and interaction effects for both randomizations). The combination of unstructured eigenconnectivities and altered dynamics suggests that real fluctuations drove the results obtained from the original data. The Hotelling's $\mathrm{T}^{2}$ combination of percentage positive weights did not correlate with EDSS.
The time-dependent weights of eigenconnectivity 10 showed a significantly increased frequency amplitude at $0.003 \mathrm{~Hz}$ in RRMS patients $(d=-1.5, \mathrm{p}<0.02$, corrected, Fig. 3 ).

We then varied the window length from short (30 s) to long (2 min) and increased step sizes from 2 to 8 TRs. Supplementary Figs. 4a-d show that while the first eigenconnectivities estimated from different window lengths roughly resemble each other, the estimated connectivity patterns are not the same. Notably, the covarying FC between the middle and inferior frontal regions apparent in eigenconnectivity 3 at a window length of 30 TRs is not detected at longer window lengths. We also observe that eigenconnectivity 5 breaks into two patterns, 4 and 6 , at a window length of 120 TRs. In general, the visible block structure of eigenconnectivities progressively vanishes at larger window lengths. The Hotelling's $\mathrm{T}^{2}$ test did not reveal significant differences between the average weights of HC subjects and RRMS patients for window lengths above 30 TRs ( $p>0.2$ ).

In exploratory work, we also repeated our analysis using different band pass filters and obtained similar results in terms of eigenconnectivities and explained variance for a high-frequency cutoff of $0.2 \mathrm{~Hz}$ and $0.3 \mathrm{~Hz}$, but no significant group difference $(\mathrm{p}=0.12$, $0.08 ; 0.64,0.75$ for group and interaction effects and the two cut-offs respectively). 


\section{Influence of intra-individual differences}

We then repeated our analysis without row-wise demeaning each subject's dynamic FC matrix but only subtracting the average dynamic FC across time and subjects. The first ten eigenconnectivities explained $42 \%$ of the variance in the data. They resembled the FC patterns estimated from the subject-wise demeaned data but were not the same (Fig. 6a). For example, the first eigenconnectivity shows much more pronounced differences between limbic and subcortical connections (more yellow than in Fig. 3), the blue connections of the 2nd one bear little resemblance, within frontal and within visual connections are less pronounced for the 3rd one, and the 6th one bears little resemblance. Eigenconnectivity 6 includes connections of the parietal regions (59-62), and the thalami $(75,76)$ with the rest of the brain, connections with visual regions (red); and the putamen $(73,74)$ to the rest of the brain and between the motor $(1,2,19,20,57,58)$ and frontal regions (blue).

The contribution of the first ten eigenconnectivities was significantly different between the two groups (group effect $F_{(10,17)}=2.7, p=0.04$,
$D=2.4$, interaction effect $\left.F_{(10,17)}=3.0, \mathrm{p}=0.02, D=2.6\right)$. The Hotelling's $T^{2}$ group difference was $\sum_{k=1}^{K} a_{k}\left(m_{k}^{H C}-m_{k}^{M S}\right)=-48 \pm$ $46 \%$, with $a_{1}=-0.76, a_{2}=0.02, a_{3}=0.03, a_{4}=-0.20, a_{5}=0.04$, $a_{6}=-0.01, a_{7}=0.30, a_{8}=-0.32, a_{9}=0.43$, and $a_{10}=-0.03$. The largest contributions thus came from the first and seventh to ninth eigenconnectivities. The percentage of positive weights for the first eigenconnectivity was $66 \pm 27 \%$ for HC subjects and $29 \pm 22 \%$ for RRMS patients $(\mathrm{p}<0.001)$. As this is an all-positive pattern, this again indicates that RRMS patients had a reduced overall FC strength. Post hoc $t$ tests also indicated a trend for eigenconnectivity 7 (61 $\pm 32 \%, 40 \pm$ $23 \%, \mathrm{p}=0.052$ ). As we did not demean each subject's dynamic FC matrix, large positive percentages can here be interpreted as reflecting stronger FC in red areas, and low percentages as reflecting stronger FC in blue areas (positive weights increase the FC of red areas, negative weights that of blue areas). Eigenconnectivity 7 contrasted connections centered on the right PCC and left Heschl gyrus (red), with connections of the limbic regions (hippocampi (HP), PAH, amygdala), the left thalamus and the left middle temporal pole (MTP, blue; Fig. 6b). The Hotelling's $\mathrm{T}^{2}$ combination of percentage positive weights did not correlate with EDSS.

\section{a) Eigenconnectivities without subject-wise demeaning}
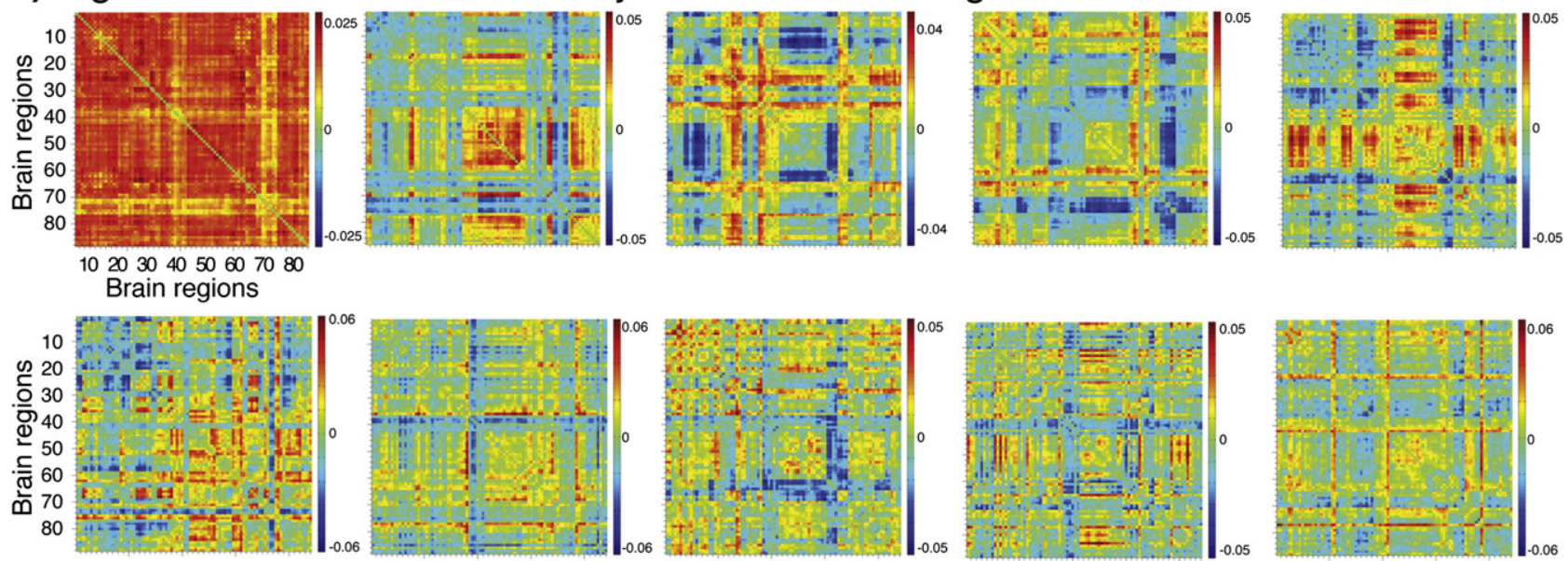

\section{b) Eigenconnectivity 7}
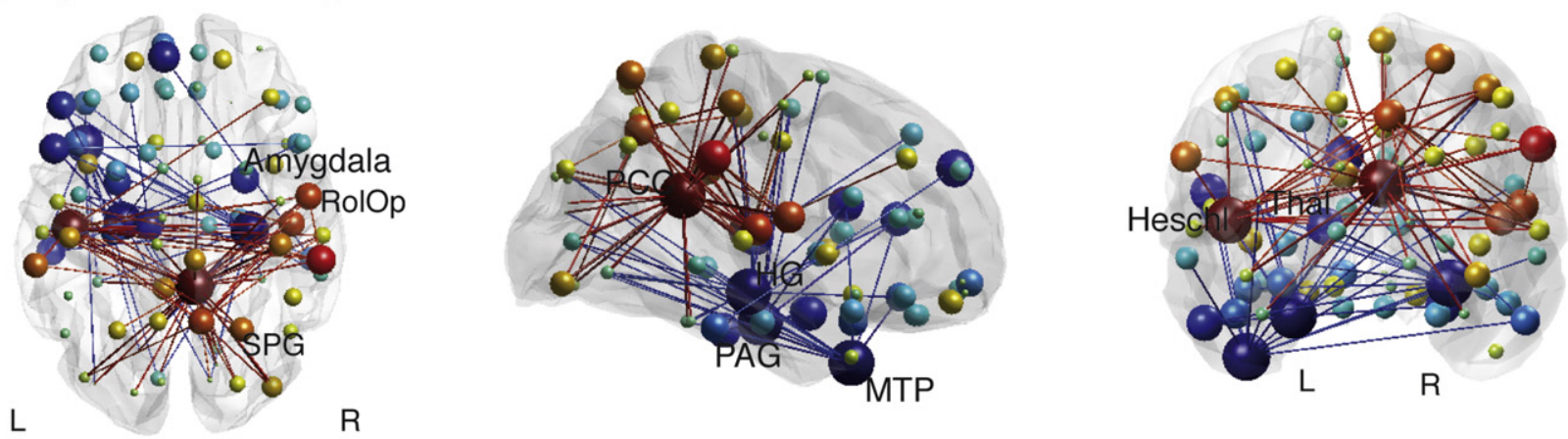

\section{c) Phase-randomized data}
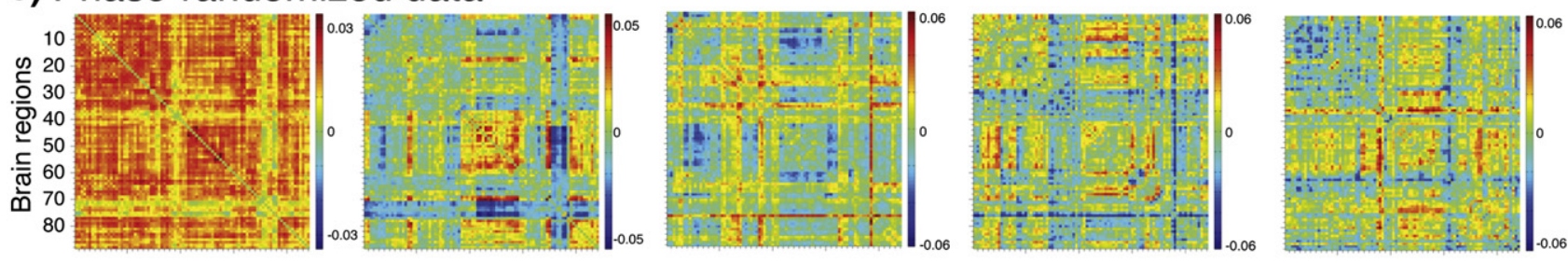

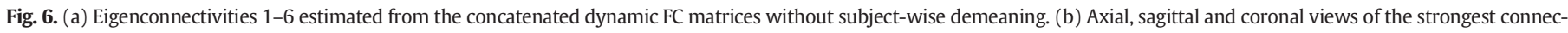

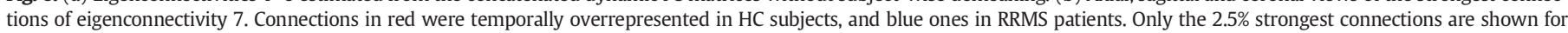
visualization purposes. (c) Eigenconnectivities estimated from phase-randomized dynamic FC matrices retain large-scale structure. 
Eigenconnectivities estimated from phase-randomized dynamic FC matrices showed structured patterns (Fig. 6c), still retained a large amount of variance ( $23 \%$ for the first ten), and the average weights differed significantly between the groups $\left(F_{(10,17)}=3.4, \mathrm{p}=0.01\right.$, $D=2.7$ for the group effect, $F_{(10,17)}=2.6, \mathrm{p}=0.04, D=2.4$ for the interaction effect). These results indicate that average correlation values rather than FC fluctuations drove the PCA and the group difference if no individual demeaning is performed.

Eigenconnectivities estimated from phase-randomized activity time courses showed no structure (Supplementary Fig. 4f), explained 5\% of variance for the first ten and Hotelling's $\mathrm{T}^{2}$ were not significant as this simulation did not preserve the mean correlation values.

\section{Motion}

Motion parameters for each subject were small (Supplementary Table 3). The four summary motion measures were not significantly different between the two groups (HC subjects and RRMS patients respectively, FD: mean 0.11, 0.11; SD 0.05, 0.05; D: mean 0.05, 0.05, SD 0.03, $0.03, p>0.4$ for all). Dynamic weights of the eigenconnectivities were not significantly correlated with any of the four motion parameters, except for two HC subjects (time-dependent weights of eigenconnectivity 4 with the standard deviation of $F D, \mathrm{R}^{2}=0.64$; time-dependent weights of eigenconnectivity 9 with the mean and standard deviation of $D, \mathrm{R}^{2}=0.75,0.73$ respectively).

As scrubbing may affect the estimation of dynamic FC, we repeated the analyses without scrubbing and found highly similar results in terms of explained variance, extracted connectivity patterns and altered contributions in MS patients.

\section{Discussion}

In this study, we introduce a novel data-driven approach for extracting dynamic FC patterns in the resting brain. This exploratory technique applies PCA to dynamic whole-brain FC of multiple subjects during resting state. PCA identified FC patterns, the eigenconnectivities, that capture FC pairs with similar dynamics. We found large-scale connectivity patterns that encompassed regions from different functional clusters. The temporal contributions of these eigenconnectivities can be obtained by projection and reveal their importance in explaining dynamic FC over time. On the example of MS, we identified a subset of connections with altered dynamics in minimally-disabled RRMS patients.

\section{Related methodological studies}

Previous studies reported large-scale reorganizations of whole-brain functional brain networks during learning (Bassett et al., 2011), task preparation (Ekman et al., 2012), or rest (Allen et al., in press). Bassett et al. (2011) studied dynamic community organization, Ekman et al. (2012) predicted upcoming tasks and errors from network measures (e.g. degree), and Allen et al. (in press) employed clustering to identify stable FC topologies. Our approach employs PCA to characterize the most abundant building blocks of dynamic FC and, like previous studies, identifies prominent changes in network topology. In contrast to "fixed" FC states detected using clustering, the eigenconnectivities overlap in time and their combined contributions give rise to varying network topologies. As such, they represent basic building blocks of network configurations that occur over time, rather than discrete states. However, clustering could also be applied to the time-dependent weights to identify eigenconnectivities with similar temporal profiles (in contrast to Allen et al. (in press) who clustered windowed correlations). We here opted for another approach and looked at summary measures of the contributions of these patterns over time.

The most prominent technique to study spontaneous brain activity is spatial ICA, which however assumes temporal stationarity. Smith et al. (2012) recently applied a cascade of spatial and temporal ICA to fast-TR data to detect functionally (temporally) distinct, spatially overlapping networks. The identified networks are static over time in terms of included voxels but their activity is dynamic. While both studies are interested in the variable architecture of the brain, the authors decomposed brain activity and not connectivity time courses in contrast to our work. As such, they identified networks of brain regions with correlated activity, whereas we identify networks of connections with correlated fluctuations. One could also consider ICA as an alternative decomposition to extract connectivity patterns from multisubject dynamic FC matrices. In the case of PCA, which optimizes for explained variance and orthogonality, we obtain an efficient, linear representation; however, determining relevant components using spatial ICA might be more difficult due to the sorting problem. Also, temporal ICA might be inappropriate for dynamic FC given its intrinsic slow dynamics.

The most prominent technique to study spontaneous brain activity and to identify voxels with similar activity is spatial ICA. To identify connections with similar fluctuations, we used PCA, which optimizes for explained variance and orthogonality, and gives an efficient, linear representation. One could also consider ICA as an alternative decomposition to extract connectivity patterns from multisubject dynamic FC matrices. It is however not clear if the assumption of statistical independence would lead to better components and, because of the independence assumption, ICA components have no inherent ordering, which would complicate the determination of relevant ones. Smith et al. (2012) recently applied a cascade of spatial and temporal ICA to fastTR data to detect functionally (temporally) distinct, and spatially overlapping networks. While both studies are interested in the architecture of the brain during rest, the authors decomposed brain activity, while we studied brain connectivity. As such, they identified networks of brain regions with similar activity, whereas we identified networks of connections with similar fluctuations. Temporal ICA might not be appropriate to decompose dynamic FC because dynamic FC fluctuates slowly due to the sliding window estimation (temporal ICA needs a large number of time points to perform robustly) and because temporal ICA implicitly assumes temporal stationarity, i.e., constant moments across time (see also Allen et al., in press; Calhoun et al., 2012, for a discussion).

\section{Stationary and dynamic FC in RRMS patients}

We identified eigenconnectivities 6 and 10 that contributed differently between HC subjects and RRMS patients, indicating aberrant dynamic FC in RRMS patients (Figs. 3f, j): The affected connections were concentrated in parietal regions such as the PCC, SPG and angular gyrus (more frequently strongly connected in RRMS patients), and prefrontal regions (e.g. orbitofrontal gyrus, gyrus rectus) and the amygdala (more frequently weakly connected). The importance of these regions suggests that the dynamic FC of at least part of the DMN is altered in patients. These results add to, and complement, several studies which have reported disturbed stationary FC in different parts of the DMN in MS patients (Bonavita et al., 2011; Hawellek et al., 2011; Richiardi et al., 2012; Rocca et al., 2012). The distinction between posterior and anterior/temporal DMN regions seen in eigenconnectivity 6 is similar to the DMN fragmentation observed in Allen et al. (in press) and would be missed in conventional stationary FC analyses.

When repeating the analysis without removing each subject's average connectivity strength, we found distinct patterns of connections that differed between HC subjects and RRMS patients, which were average- rather than dynamically-driven (Fig. 6). The identified brain regions are largely consistent with those in a previous study by our group, which aimed at discriminating between HC subjects and RRMS patients based on stationary FC (Richiardi et al., 2012); i.e., the left Heschl's gyrus, right rolandic operculum, and right superior parietal gyrus, were identified as being more strongly connected in HC subjects 
in both studies. Reduced stationary FC in MS patients is indeed commonly observed and is thought to stem from structural damage (Bonavita et al., 2011; Lowe et al., 2002; Richiardi et al., 2012; Roosendaal et al., 2010). However, we also confirmed the bilateral parahippocampal gyri, right amygdala, left thalamus and left midtemporal pole as more strongly connected in RRMS patients (Richiardi et al., 2012). Such selective increased FC has been reported by several other studies as well (Bonavita et al., 2011; Dogonowski et al., 2013; Hawellek et al., 2011; Richiardi et al., 2012; Rocca et al., 2012; Roosendaal et al., 2010) and has been interpreted as a compensatory mechanism (Bonavita et al., 2011; Roosendaal et al., 2010) because activation studies have shown increased activity in regions devoted to the task and the recruitment of additional regions in MS patients (Pantano et al., 2002; Rocca et al., 2005). It is however also possible that these increases originate from a loss of flexibility in functional interactions, rather than as a compensatory mechanism (Hawellek et al., 2011). Interestingly, the connections in eigenconnectivity 10 obtained from the dynamic FC analysis showed an increased low-frequency amplitude in RRMS patients, which might support the latter hypothesis.

While we did not observe correlations between the Hotelling's $\mathrm{T}^{2}$ combinations of positive percentages and clinical disability for the dynamically- or average-driven analysis, it should be noted that our sample size was relatively small (15 patients), and our patients minimally disabled (EDSS range 1.5-2.5, meaning that they can live a normal life). It would be interesting to study changes in dynamic FC in larger samples of MS patients, at different phases of the disease, and using additional clinical measures. Nonetheless, given the complementary insights gained from the analysis of dynamic FC, such as which connections fluctuate coherently or which connections fluctuate more slowly, we believe that studies of dynamic FC can help in understanding the functional impact of MS. However, we note that the choice of sliding window length and band pass filter affected our results as opposed to scrubbing or the removal of small weights. Therefore, while our results are promising, studies in independent cohorts are needed to further explore these effects and validate our findings.

\section{Timescales and potential confounds of dynamic FC}

Correlations estimated from short windows have more variance due to the small number of samples but are more sensitive to changes in FC since long windows reduce temporal variability and may miss anticorrelations (Chang and Glover, 2010; Hutchison et al., in press). Some studies have reported that $30 \mathrm{~s}$ of data suffices to discriminate between cognitive states and to estimate reliable modular graph metrics (Jones et al., 2012; Shirer et al., 2012). We therefore chose a sliding window length of 30 TRs to capture changes in FC over time. Given the similarities between estimated eigenconnectivities at different window lengths, it seems unlikely that it is random variability that drove FC patterns at short window lengths. Exploratory work using different band pass cut-off frequencies also suggested that the large-scale structure of the eigenconnectivities was largely preserved across broad frequency ranges. In our main analysis we chose a cut-off of $0.15 \mathrm{~Hz}$ as sliding window correlations are estimated from a small number of samples and a lower cut-off helps to remove noise. The chosen cut-off is similar to what has been used in other studies on dynamic FC (Allen et al., in press; Bassett et al., 2011; Handwerker et al., 2012; Kang et al., 2011; Li et al., in press), but it would be interesting to further investigate the effects of different frequency cut-offs on dynamic FC when physiological data is available to reduce noise in resting-state activity time courses. Together, these results suggest that patterns of covarying connections were largely similar across varying window lengths, frequency cutoffs and with or without scrubbing.

We also tested for correlations between the contributions of the eigenconnectivities across time and motion measures. Out of the 28 subjects, these contributions were only significantly correlated for two subjects and one of the eigenconnectivities. While we cannot exclude that motion-related or physiological noise is potentially driving the observed FC patterns, the large-scale organization of these patterns, the importance of the precise timing of FC fluctuations as shown by the results with phase-randomized dynamic FC and the minimal correlations with motion parameters, suggest that these patterns are not simply the result of noise.

Chang et al. (2013b) recently linked heart rate variability to dynamic FC in regions associated with vigilance and arousal, suggesting the contribution of autonomic factors to the observed nonstationarity. Chang and colleagues also showed that alpha band power in EEG was related to FC fluctuations between the DMN and the dorsal attention network (Chang et al., 2013a). Further concurrent EEG-fMRI studies will be crucial in clarifying the relationship between these fluctuations and neural dynamics, as well as how they relate to attentional states. Along those lines, the scale-free dynamics of EEG microstate sequences have been shown to reach timescales of fMRI resting-state fluctuations (Van de Ville et al., 2010) and the link between EEG microstates and dynamic fMRI FC is an intriguing future research question.

\section{Variability as a signal in its own right}

Not only has interest in the variability of brain connectivity increased, but several recent studies have also highlighted that the variability of brain activity is a signal of interest and provides information complementary to the analysis of mean brain activity. Notably, brain activity variability has been linked to task performance, development and disease and it has been suggested that signal variability is not "noise" but crucial for spontaneous or stimulated reorganizations of the brain (Garrett et al., 2010; McIntosh et al., 2008). Just as studies on brain signal variability provided novel insights into brain function, we believe that studies on brain connectivity variability will further our understanding of the brain's functional organization. Calhoun and Adali (2012) and Starck et al. (2012) recently reported changes in dynamic FC variability in patients with schizophrenia and autism. We believe that our proposed approach can go beyond identifying changes in dynamic FC variability and reveal patterns in FC fluctuations. Our approach is also applicable to other neurodegenerative diseases and the temporal evolution of the brain's functional network at longer time scales, such as during learning or development.

\section{Methodological limitations and future directions}

In this study we used anatomically defined regions and a simple linear estimator of dynamic FC, but other parcellations or more complex estimators of dynamic FC could easily be used with the proposed approach.

A comparison between the real eigenvalues and those obtained from the phase-randomized data suggested that the first 30 components predominantly carried signal, while the latter ones were not statistically different from noise. As the eigenvalues were close in magnitude, it seems however reasonable to not take this number too literally but rather as an indication of the overall range. An alternative method to estimate the dimensionality of our data, Laplace PCA (Minka, 2000), grossly overestimated its dimensionality (1362 dimensions). The comparison of the eigenvalues to those obtained from surrogate data on the other hand provided a reasonable, rough estimate to guide the choice of dimensionality. Other dimensionality estimation approaches, such as those used in activation studies (Yourganov et al., 2011) or ICA (Varoquaux et al., 2010), could be adapted to dynamic FC and evaluated in future studies. The ten first eigenconnectivities used for further analysis did not use the full "signal space" and explained 34\% of the variance in dynamic FC, but our results suggest that they captured important differences between the groups. To identify connectivity patterns across subjects (rather than modeling subject-specific patterns or noise), it might indeed not be necessary to explain a majority of the variance. This is also highlighted by recent work of Li et al. (in press) 
who identified 16 FC states using a dictionary learning approach and whose optimal model had residuals of around $50 \%$.

It should also be noted that PCA is a linear decomposition and only sensitive to linear relations among connectivity pairs. To identify FC patterns based on nonlinear relations, one could for example employ kernel PCA, an extension of PCA, which has shown promising results for face recognition (Schölkopf et al., 1998).

Finally, we also mention that other measures should be explored to exploit the full potential of temporal dynamics of eigenconnectivity time-dependent weights, in addition to the proposed percentage of positive weights.

In conclusion, we proposed a novel approach to estimate building blocks of dynamic brain connectivity and assessed their disturbance in minimally disabled RRMS patients.

\section{Acknowledgments}

This work was supported by the Swiss National Science Foundation (grant numbers PP0OP2-123438 and PP00P2-146318), the Sociéte Académique de Genève (FOREMANE fund), the Swiss Society for Multiple Sclerosis, the Merck-Serono EPFL Research Alliance Award, the Center for Biomedical Imaging (CIBM) of the Geneva and Lausanne Universities, the EPFL, and the Leenaards and Louis-Jeantet Foundations. JR was supported by a Marie Curie International Outgoing Fellowship from the EU (MC IOF 299500). We thank M. Leonardi for the photograph of the eigenconnectivity cubes.

\section{Conflict of interest}

The authors declare no conflict of interest.

\section{Appendix A. Supplementary data}

Supplementary data to this article can be found online at http://dx. doi.org/10.1016/j.neuroimage.2013.07.019.

\section{References}

Allen, E.A., Damaraju, E., Plis, S.M., Erhardt, E.B., Eichele, T., Calhoun, V.D., 2013. Tracking whole-brain connectivity dynamics in the resting state. Cereb. Cortex (in press).

Ashburner, J., Friston, K.J., 2005. Unified segmentation. NeuroImage 26, 839-851.

Barkhof, F., 2002. The clinico-radiological paradox in multiple sclerosis revisited. Curr. Opin. Neurol. 15, 239-245.

Bassett, D., Wymbs, N., Porter, M., Mucha, P., Carlson, J., Grafton, S., 2011. Dynamic reconfiguration of human brain networks during learning. Proc. Natl. Acad. Sci. U. S. A $108,7641-7646$.

Beckmann, C.F., Mackay, C.E., Filippini, N., Smith, S.M., 2009. Group Comparison of Resting-State fMRI Data Using Multi-Subject ICA and Dual Regression. OHBM, p. 1-1.

Biswal, B., Yetkin, F.Z., Haughton, V.M., Hyde, J.S., 1995. Functional connectivity in the motor cortex of resting human brain using echo-planar MRI. Magn. Reson. Med. 34, 537-541.

Bjornsson, H., Venegas, S.A., 1997. A Manual for EOF and SVD Analyses of Climatic Data.

Bonavita, S., Gallo, A., Sacco, R., Corte, M., Bisecco, A., Docimo, R., Lavorgna, L., Corbo, D., D Costanzo, A., Tortora, F., Cirillo, M., Esposito, F., Tedeschi, G., 2011. Distributed changes in default-mode resting-state connectivity in multiple sclerosis. Mult. Scler. 17, 411-422.

Britz, J., Van De Ville, D., Michel, C.M., 2010. BOLD correlates of EEG topography reveal rapid resting-state network dynamics. NeuroImage 52, 1162-1170.

Calhoun, V., Adali, T., 2012. Multi-subject independent component analysis of fMRI: a decade of intrinsic networks, default mode, and neurodiagnostic discovery. IEEE Rev. Biomed. Eng. 5, 60-73.

Calhoun, V.D., Eichele, T., Adal, T., Allen, E.A., 2012. Decomposing the brain: components and modes, networks and nodes. Trends Cogn. Sci. 16, 255-256.

Chang, C., Glover, G., 2010. Time-frequency dynamics of resting-state brain connectivity measured with fMRI. NeuroImage 50, 81-98.

Chang, C., Liu, Z., Chen, M.C., Liu, X., Duyn, J.H., 2013a. EEG correlates of time-varying BOLD functional connectivity. Neurolmage 72, 227-236.

Chang, C., Metzger, C.D., Glover, G.H., Duyn, J.H., Heinze, H.J., Walter, M., 2013b. Association between heart rate variability and fluctuations in resting-state functional connectivity. Neurolmage 68, 93-104.

Chao-Gan, Y., Yu-Feng, Z., 2010. DPARSF: a MATLAB toolbox for "pipeline" data analysis of resting-state fMRI. Front. Syst. Neurosci. 4, 13.

Compston, A., Coles, A., 2008. Multiple sclerosis. Lancet 372, 1502-1517.
Cordes, D., Nandy, R.R., 2006. Estimation of the intrinsic dimensionality of fMRI data. Neurolmage 29, 145-154.

Cribben, I., Haraldsdottir, R., Atlas, L.Y., Wager, T.D., Lindquist, M.A., 2012. Dynamic connectivity regression: determining state-related changes in brain connectivity. Neurolmage 61, 907-920.

Damoiseaux, J.S., Rombouts, S.A.R.B., Barkhof, F., Scheltens, P., Stam, C.J., Smith, S.M., Beckmann, C.F., 2006. Consistent resting-state networks across healthy subjects. Proc. Natl. Acad. Sci. U. S. A. 103, 13848-13853.

Dogonowski, A.M., Siebner, H.R., Srensen, P.S., Wu, X., Biswal, B., Paulson, O.B., Dyrby, T.B., Skimminge, A., Blinkenberg, M., Madsen, K.H., 2013. Expanded functional coupling of subcortical nuclei with the motor resting-state network in multiple sclerosis. Mult. Scler. 19, 559-566.

Ekman, M., Derrfuss, J., Tittgemeyer, M., Fiebach, C.J., 2012. Predicting errors from reconfiguration patterns in human brain networks. Proc. Natl. Acad. Sci. U. S. A. 109, 16714-16719.

Eryilmaz, H., Ville, D.V.D., Schwartz, S., Vuilleumier, P., 2011. Impact of transient emotions on functional connectivity during subsequent resting state: a wavelet correlation approach. Neurolmage 54, 2481-2491.

Filippi, M., Agosta, F., 2010. Imaging biomarkers in multiple sclerosis. J. Magn. Reson. Imaging 31, 770-788.

Fox, M.D., Greicius, M., 2010. Clinical applications of resting state functional connectivity. Front. Syst. Neurosci. 4,19.

Fox, M.D., Raichle, M.E., 2007. Spontaneous fluctuations in brain activity observed with functional magnetic resonance imaging. Nat. Rev. Neurosci. 8, 700-711.

Friston, K.J., Frith, C.D., Liddle, P.F., Frackowiak, R.S., 1993. Functional connectivity: the principal-component analysis of large (PET) data sets. J. Cereb. Blood Flow Metab. $13,5-14$.

Friston, K.J., Jezzard, P., Frackowiak, R.S.J., Turner, R., 1994. Functional MRI of the brain. chapter Characterizing Focal and Distributed Physiological Changes With MRI and PET. Society of Magnetic Resonance in Medicine, Berkeley, CA, pp. 207-216.

Fu, L., Matthews, P.M., De Stefano, N., Worsley, K.J., Narayanan, S., Francis, G.S., Antel, J.P., Wolfson, C., Arnold, D.L., 1998. Imaging axonal damage of normal-appearing white matter in multiple sclerosis. Brain 121, 103-113.

Garrett, D.D., Kovacevic, N., McIntosh, A.R., Grady, C.L., 2010. Blood oxygen leveldependent signal variability is more than just noise. J. Neurosci. 30, 4914-4921.

Hackmack, K., Paul, F., Weygandt, M., Allefeld, C., Haynes, J.D., Initiative, A.D.N., 2012. Multi-scale classification of disease using structural MRI and wavelet transform. Neurolmage 62, 48-58.

Handwerker, D.A., Roopchansingh, V., Gonzalez-Castillo, J., Bandettini, P.A., 2012. Periodic changes in fMRI connectivity. NeuroImage 63, 1712-1719.

Hawellek, D.J., Hipp, J.F., Lewis, C.M., Corbetta, M., Engel, A.K., 2011. Increased functional connectivity indicates the severity of cognitive impairment in multiple sclerosis. Proc. Natl. Acad. Sci. U. S. A. 108, 19066-19071.

Hutchison, R.M., Womelsdorf, T., Gati, J.S., Everling, S., Menon, R.S., 2013. Resting-state networks show dynamic functional connectivity in awake humans and anesthetized macaques. Hum. Brain Mapp. (in press).

Jones, D.T., Vemuri, P., Murphy, M.C., Gunter, J.L., Senjem, M.L., Machulda, M.M., Przybelski, S.A., Gregg, B.E., Kantarci, K., Knopman, D.S., Boeve, B.F., Petersen, R.C., Jack Jr., C.R., 2012. Non-stationarity in the "resting brain's" modular architecture. PLoS One 7, e39731.

Kang, J., Wang, L., Yan, C., Wang, J., Liang, X., He, Y., 2011. Characterizing dynamic functional connectivity in the resting brain using variable parameter regression and Kalman filtering approaches. NeuroImage 56, 1222-1234.

Kitzbichler, M.G., Smith, M.L., Christensen, S.R., Bullmore, E., 2009. Broadband criticality of human brain network synchronization. PLoS Comput. Biol. 5, e1000314.

Kiviniemi, V., Kantola, J.H., Jauhiainen, J., Hyvrinen, A., Tervonen, O., 2003. Independent component analysis of nondeterministic fMRI signal sources. Neurolmage 19, 253-260.

Kiviniemi, V., Vire, T., Remes, J., Elseoud, A.A., Starck, T., Tervonen, O., Nikkinen, J., 2011. A sliding time-window ICA reveals spatial variability of the default mode network in time. Brain Connect. 1, 339-347.

Krishnan, A., Williams, L.J., McIntosh, A.R., Abdi, H., 2011. Partial Least Squares (PLS) methods for neuroimaging: a tutorial and review. NeuroImage 56, 455-475.

Kurtzke, J.F., 1983. Rating neurologic impairment in multiple sclerosis: an expanded disability status scale (EDSS). Neurology 33, 1444-1452.

Laird, A.R., Fox, P.M., Eickhoff, S.B., Turner, J.A., Ray, K.L., McKay, D.R., Glahn, D.C., Beckmann, C.F., Smith, S.M., Fox, P.T., 2011. Behavioral interpretations of intrinsic connectivity networks. J. Cogn. Neurosci. 23, 4022-4037.

Larson-Prior, L.J., Zempel, J.M., Nolan, T.S., Prior, F.W., Snyder, A.Z., Raichle, M.E., 2009. Cortical network functional connectivity in the descent to sleep. Proc. Natl. Acad. Sci. U. S. A. 106, 4489-4494.

Li, X., Zhu, D., Jiang, X., Jin, C., Zhang, X., Guo, L., Zhang, J., Hu, X., Li, L., Liu, T., 2013. Dynamic functional connectomics signatures for characterization and differentiation of PTSD patients. Hum. Brain Mapp. (in press).

Lowe, M.J., Phillips, M.D., Lurito, J.T., Mattson, D., Dzemidzic, M., Mathews, V.P., 2002. Multiple sclerosis: low-frequency temporal blood oxygen level-dependent fluctuations indicate reduced functional connectivity initial results. Radiology 224, 184-192.

Majeed, W., Magnuson, M., Hasenkamp, W., Schwarb, H., Schumacher, E.H., Barsalou, L., Keilholz, S.D., 2011. Spatiotemporal dynamics of low frequency BOLD fluctuations in rats and humans. NeuroImage 54, 1140-1150.

McIntosh, A.R., Bookstein, F.L., Haxby, J.V., Grady, C.L., 1996. Spatial pattern analysis of functional brain images using partial least squares. Neurolmage 3, 143-157.

McIntosh, A.R., Kovacevic, N., Itier, R.J., 2008. Increased brain signal variability accompanies lower behavioral variability in development. PLoS Comput. Biol. 4, e1000106.

McKeown, M.J., Makeig, S., Brown, G.G., Jung, T.P., Kindermann, S.S., Bell, A.J., Sejnowski, T.J., 1998. Analysis of fMRI data by blind separation into independent spatial components. Hum. Brain Mapp. 6, 160-188. 
Minka, T.P., 2000. Automatic choice of dimensionality for PCA. NIPS, pp. 598-604.

Nichols, T.E., Holmes, A.P., 2002. Nonparametric permutation tests for functional neuroimaging: a primer with examples. Hum. Brain Mapp. 15, 1-25.

Pantano, P., Iannetti, G.D., Caramia, F., Mainero, C., Di Legge, S., Bozzao, L., Pozzilli, C., Lenzi, G.L., 2002. Cortical motor reorganization after a single clinical attack of multiple sclerosis. Brain 125, 1607-1615.

Pascual-Marqui, R.D., Michel, C.M., Lehmann, D., 1995. Segmentation of brain electrical activity into microstates: model estimation and validation. IEEE Trans. Biomed. Eng. 42, 658-665.

Polman, C.H., Reingold, S.C., Edan, G., Filippi, M., Hartung, H.P., Kappos, L., Lublin, F.D., Metz, L.M., McFarland, H.F., O'Connor, P.W., Sandberg-Wollheim, M., Thompson, A.J., Weinshenker, B.G., Wolinsky, J.S., 2005. Diagnostic criteria for multiple sclerosis: 2005 revisions to the "McDonald Criteria". Ann. Neurol. 58, 840-846.

Power, J.D., Barnes, K.A., Snyder, A.Z., Schlaggar, B.L., Petersen, S.E., 2012. Spurious but systematic correlations in functional connectivity MRI networks arise from subject motion. Neurolmage 59, 2142-2154.

Richiardi, J., Eryilmaz, H., Schwartz, S., Vuilleumier, P., Van De Ville, D., 2011. Decoding brain states from fMRI connectivity graphs. NeuroImage 56, 616-626.

Richiardi, J., Gschwind, M., Simioni, S., Annoni, J.M., Greco, B., Hagmann, P., Schluep, M., Vuilleumier, P., Van De Ville, D., 2012. Classifying minimally disabled multiple sclerosis patients from resting state functional connectivity. Neurolmage 62, 2021-2033.

Rocca, M.A., Colombo, B., Falini, A., Ghezzi, A., Martinelli, V., Scotti, G., Comi, G., Filippi, M. 2005. Cortical adaptation in patients with MS: a cross-sectional functional MRI study of disease phenotypes. Lancet Neurol. 4, 618-626.

Rocca, M.A., Valsasina, P., Absinta, M., Riccitelli, G., Rodegher, M.E., Misci, P., Rossi, P., Falini, A., Comi, G., Filippi, M., 2010. Default-mode network dysfunction and cognitive impairment in progressive MS. Neurology 74, 1252-1259.

Rocca, M.A., Valsasina, P., Martinelli, V., Misci, P., Falini, A., Comi, G., Filippi, M., 2012. Large-scale neuronal network dysfunction in relapsing-remitting multiple sclerosis. Neurology 79, 1449-1457.

Roosendaal, S.D., Schoonheim, M.M., Hulst, H.E., Sanz-Arigita, E.J., Smith, S.M., Geurts, J.J.G., Barkhof, F., 2010. Resting state networks change in clinically isolated syndrome. Brain 133, 1612-1621.
Schölkopf, B., Smola, A., Müller, K.R., 1998. Nonlinear component analysis as a kernel eigenvalue problem. Neural Comput. 10, 1299-1319.

Schoonheim, M.M., Filippi, M., 2012. Functional plasticity in MS: friend or foe? Neurology 79, 1418-1419.

Shirer, W.R., Ryali, S., Rykhlevskaia, E., Menon, V., Greicius, M.D., 2012. Decoding subjectdriven cognitive states with whole-brain connectivity patterns. Cereb. Cortex 22, $158-165$.

Smith, S.M., Fox, P.T., Miller, K.L., Glahn, D.C., Fox, P.M., Mackay, C.E., Filippini, N., Watkins, K.E., Toro, R., Laird, A.R., Beckmann, C.F., 2009. Correspondence of the brain's functional architecture during activation and rest. Proc. Natl. Acad. Sci. U. S. A. 106, 13040-13045.

Smith, S.M., Miller, K.L., Moeller, S., Xu, J., Auerbach, E.J., Woolrich, M.W., Beckmann, C.F. Jenkinson, M., Andersson, J., Glasser, M.F., Van Essen, D.C., Feinberg, D.A., Yacoub, E.S., Ugurbil, K., 2012. Temporally-independent functional modes of spontaneous brain activity. Proc. Natl. Acad. Sci. U. S. A. 109, 3131-3136.

Starck, T., Nikkinen, J., Remes, J., Rahko, J., Moilanen, I., Tervonen, O., Kiviniemi, V., 2012. Temporally Varying Connectivity Between ICA Default-Mode Sub-Networks - ASD vs. Controls. OHBM, p. 1-1.

Theiler, J., Eubank, S., Longtin, A., Galdrikian, B., Farmer, J.D., 1992. Testing for nonlinearity in time series: the method of surrogate data. Physica D 58, 77-94.

Tzourio-Mazoyer, N., Landeau, B., Papathanassiou, D., Crivello, F., Etard, O., Delcroix, N., Mazoyer, B., Joliot, M., 2002. Automated anatomical labeling of activations in SPM using a macroscopic anatomical parcellation of the MNI MRI single-subject brain. Neurolmage 15, 273-289.

Van de Ville, D., Britz, J., Michel, C.M., 2010. EEG microstate sequences in healthy humans at rest reveal scale-free dynamics. Proc. Natl. Acad. Sci. U. S. A. 107, 18179-18184.

Varoquaux, G., Sadaghiani, S., Pinel, P., Kleinschmidt, A., Poline, J.B., Thirion, B., 2010. A group model for stable multi-subject ICA on fMRI datasets. Neurolmage 51, 288-299.

Vincent, J.L., Patel, G.H., Fox, M.D., Snyder, A.Z., Baker, J.T., Van Essen, D.C., Zempel, J.M., Snyder, L.H., Corbetta, M., Raichle, M.E., 2007. Intrinsic functional architecture in the anaesthetized monkey brain. Nature 447, 83-86.

Yourganov, G., Chen, X., Lukic, A.S., Grady, C.L., Small, S.L., Wernick, M.N., Strother, S.C., 2011. Dimensionality estimation for optimal detection of functional networks in BOLD fMRI data. NeuroImage 56, 531-543. 